\title{
Are Interest Rate Derivatives Spanned by the Term Structure of Interest Rates?
}

\author{
Massoud Heidari \\ Caspian Capital Management \\ Liuren $\mathrm{Wu}$ \\ Fordham University
}

Follow this and additional works at: https://fordham.bepress.com/crif_working_papers

Part of the Finance and Financial Management Commons

\section{Recommended Citation}

Heidari, Massoud and Wu, Liuren, "Are Interest Rate Derivatives Spanned by the Term Structure of Interest Rates?" (2001). CRIF Working Paper series. 25.

https://fordham.bepress.com/crif_working_papers/25 


\title{
Are Interest Rate Derivatives Spanned by the Term Structure of Interest Rates?*
}

\author{
Massoud HeIDARI ${ }^{\dagger}$ \\ Caspian Capital Management, $L L C$ \\ LIUREN WU $\mathrm{U}^{\ddagger}$ \\ Graduate School of Business, Fordham University
}

September 20, 2001; first draft: July 11, 2000

\footnotetext{
*We thank Ayman Hindy, Chi-fun Huang, and William Dellal for numerous detailed discussions and helpful comments. We thank Lehman Brothers for providing the data. We also thank Maurice Chen, Silverio Foresi, Karl Kolderup, Markus Leippold, Enlin Pan, and Tong Yao for helpful suggestions. We welcome comments, including references to related papers we have inadvertently overlooked. The latest version of the paper can be downloaded from http://www. bnet.fordham.edu/lwu.

${ }^{\dagger}$ Correspondence Information: 1251 Avenue of the Americas, 16th floor, New York, NY 10020; tel: (212) 703-0333; fax: (212) 703-0380; Massoud.Heidari@caspiancapital.com.

${ }^{\ddagger}$ Correspondence Information: 113 West 60th Street, New York, NY 10023; tel: (212) 636-6117; fax: (212)765-5573; wu@fordham.edu.
} 


\title{
Are Interest Rate Derivatives Spanned by the Term Structure of Interest Rates?
}

\begin{abstract}
We investigate whether the same finite dimensional dynamic system spans both interest rates (the yield curve) and interest rate options (the implied volatility surface). We find that the options market exhibits factors independent of the underlying yield curve. While three common factors are adequate to capture the systematic movement of the yield curve, we need three additional factors to capture the movement of the implied volatility surface.
\end{abstract}

JeL Classification Codes: E43, G12.

KEY WORDS: Factors; principal component; LIBOR; swaps; swaptions; yield curve; implied volatility surface. 
We measure and interpret common factors underlying the US dollar LIBOR market that includes both interest rates and interest rate options. In particular, we investigate whether the same finite dimensional dynamic system spans both types of instruments. We find that the options market exhibits factors independent of the underlying yield curve. We identify three common factors from LIBOR and swap rates; yet, these three factors can only explain half of the variation in the swaption implied volatilities. We need three additional factors to capture the movement of the implied volatility surface.

Our interest in the issue stems from our observation that two groups of practitioners in the fixed income market take sharply different approaches in applying interest rate models. For fund managers trading the yield curve, the approach is quite similar to that of the academics who work on equilibrium or structural models. They choose a two or three factor structural model, estimate the model parameters from the time series data, and then at each day, choose the level of the state variables (factors) to fit the current term structure at a finite number of maturities. The discrepancies between the fitted term structure and the market prices are perceived as potential trading opportunities. On the other hand, interest rate option traders often take the yield curve as given with minimal or no smoothing. To fit the observed yield curve perfectly, they often allow some of the model parameters to be time-inhomogeneous. The model is then solved based on no-arbitrage conditions as in Heath, Jarrow, and Morton (1992), assuming some term structure for the volatilities. Even so, they often need to recalibrate the model every day, changing the parameters to match the newly observed implied volatility surface.

The objective of this paper is to infer important information about the interest rate market from such divergent practices, rather than discussing the limitations of the structural models or the inconsistencies of the no-arbitrage approach. The fact that yield-curve traders apply a lowdimensional structural model implies that a finite set of state variables are sufficient to capture most of the common variations on the yield curve. The idiosyncratic part is potentially due to transient technical dislocations. On the other hand, the "full yield curve fitting" practice in the option market implies that option traders do not want to be exposed to the idiosyncratic risk in the interest rate market, potentially because they have their own "independent" risks 
to deal with. Taking the yield curve as given assumes as many state variables as the interest rate quotes; changing model parameters daily means that the parameters are also variables. Therefore, quite possibly, the structural models are limited only when applied to the options market; the no-arbitrage approach is applied inconsistently only because the option traders cannot conceive a consistent way to capture the variations of the options market with the same yield curve factors.

Our empirical findings confirm such conjectures. Similar to Litterman and Scheinkman (1991), we identify three common interest rate factors which represent the level, the slope, and the curvature of the yield curve, respectively. The three factors explain $99.50 \%$ of the variation on the yield curve. However, when applied to swaption implied volatilities, these three common factors only explain $59.48 \%$ of the variation in that market. To adequately explain the variation in the implied volatility surface, we need three additional volatility factors. These volatility factors are independent of the interest rate movement, but are crucial in explaining the movement of interest rate options. Together with the three interest rate factors, they explain $97.62 \%$ of the variation on the implied volatility surface.

Furthermore, we find that the three volatility factors mainly capture the independent variation of the implied volatility surface along the option expiry dimension. Analogous to the roles played by the three interest rate factors on the yield curve, the three volatility factors capture, respectively, the level, the slope, and the curvature of the option expiry term structure of the implied volatility surface. Variations of the surface along the swap maturity dimension are mostly captured by the interest rate factors, but are relatively insensitive to the volatility factors.

Our findings have important implications for pricing and hedging in the fixed income market. In theory, in an efficient market, the yield curve and the implied volatility surface should be driven by the same set of factors. Therefore, the identification of independent volatility factors is intriguing. One potential explanation for the independent factors is that the swaptions market is relatively inefficient compared to the interest rate market. If this is the case, one can potentially design trading strategies to exploit such inefficiencies. Another related interpretation is that the two markets are partially segmented so that cross-market arbitrage 
strategies are difficult to implement. Thus, even though the practices of players in the two markets are inconsistent with each other, they may still be relatively "safe" within their own respective market. However, such "safe" practices will break down for fund managers investing in both markets. In reality, the two markets are inherently linked through fixed income securities with embedded options. For example, a large percentage of corporate debt has callable and/or puttable features. Even more importantly, the single largest and fastest growing fixed income category in the United States is mortgage backed securities. The prepayment options embedded in the mortgage market force the fund managers to face and hence manage risks from both the interest rate market and the options market simultaneously. Since these securities cross both markets, it is imperative to reconcile the potential inconsistencies between the two approaches in pricing and hedging.

On the other hand, the finding of independent factors may simply be revealing a certain model structure, under which some of the factors have statistically negligible impacts on the yield curve, but have significant impacts on option prices. In this case, the "independent" factors are not exactly independent of the yield curve in theory, but the dependence exhibits no statistical or economic significance. If one can identify such a term structure model, one can calibrate the three common factors on the yield curve, safely pretending that the additional volatility factors do not exist, and then proceed to calibrate the volatility factors only to the swaption implied volatility surface. Identifying such a model structure not only solves the consistency issue between the two markets, but also implies significant efficiency gains in model calibration. We label such a model structure as an $m+n$ model, where $m$ denotes the number of common factors and $n$ denotes the number of independent volatility factors. If such a model structure exists, our empirical results point to a $3+3$ structure.

The existence and identification of a finite number of factors is also vitally important for hedging practices. In the absence of a finite dimensional representation for the yield curve, even if the options market is spanned by the interest rates, holding the theoretical hedging portfolio including an infinite number of interest rate instruments becomes infeasible in practice due to transaction costs. Daily adjustment of an infinite number of instruments leads to ruin given the existence of any transaction costs. Our identification of three interest rate factors 
implies that the yield curve movement can be hedged by merely three instruments. Hedging swaptions, on the other hand, requires three additional option contracts which capture the movement of the level, slope, and curvature of the implied volatility surface along the option maturity dimension.

To ensure robust results for our analysis, we pay special attention to details in data choice and methodologies. For data choice, we choose to analyze the US dollar LIBOR market instead of US Treasuries. The most important reason for such a choice is that the swap contracts do not suffer from the problems induced by supply and demand for US Treasuries. Since 1997, the outstanding Treasury supply has been dropping due to the growing budget surplus. The reduced supply has already raised the possibility of squeezes, which have shown up in the abnormal differences in yields between swaps and Treasuries, e.g. Masson (2000). The short maturity Treasury bills have also been found to exhibit idiosyncratic behaviors due to, among other things, their different margin equivalence from Treasury bonds. See, for example, Duffee (1996), Knez, Litterman, and Scheinkman (1994), and Longstaff (2000). On the other hand, the credit risk of swap contracts are in general very small due to their two-way mark-to-market nature, e.g. He (2000) and Liu, Longstaff, and Mandell (2000). As a result, LIBOR and swap rates are replacing Treasury rates as benchmarks in the industry for pricing fixed income securities.

In contrast to traditional practices in factor analysis, rather than computing excess returns, we perform the analysis directly on the LIBOR and swap rate quotes and implied volatility quotes. To compute excess returns from the finite points of observations, we would be forced to apply smoothing and interpolation. Yet, recently, Björk and Christensen (1999) and Filipović (1999, 2000) have shown that the commonly used smoothing functions, such as the NelsonSiegel family and cubic spline, are not consistent with any diffusion dynamics. We do not interpolate the data and thus avoid any spurious effects induced by such practice.

Furthermore, in spite of the high persistence in interest rates and implied volatilities, we choose to perform the factor analysis on the levels, rather than their first differences. The choice is based on our conjecture that the idiosyncratic risk is much less persistent than the common factors. We illustrate that, under such a conjecture, taking differences artificially 
deflates the relative significance of information versus noise and hence reduces the efficiency of the estimation. The conjecture is based on the well-documented evidence of cointegration in interest rates, ${ }^{1}$ e.g. Alexander and Johnson (1994), Bauwens, Deprins, and Vandeuren (1997), Bradley and Lumpkin (1992), Campbell and Shiller (1987), Engle and Granger (1987), Hall, Anderson, and Granger (1992), and Shiller (1981). Analysis of the estimated factors and residuals confirms our conjecture of reduced serial correlation in residuals. For robustness, we repeat our analysis on first differences at several frequencies and confirm that taking differences dramatically reduces the estimation efficiency.

Finally, in determining the optimal number of factors, we recognize the sharply different liquidity features between the swap market and the swaption market. In particular, we propose a modified version of the test statistic in Connor and Korajczyk (1993) which takes explicit account of the large difference in bid-ask spreads between the two markets. While the adjustment has little impact on the more liquid interest rate market, it significantly reduces the optimal number of factors needed for the less liquid swaption market.

This article extends the classic works by Litterman and Scheinkman (1991) and Knez, Litterman, and Scheinkman (1994), who perform factor analysis on the US Treasury bond market in the former and the money market in the latter. Their works have become the standard reference for both model designs and hedging practices. In this article, we extend their analysis to include interest rate options. The objective is to provide guidance for future endeavors in model design and risk management which cover both interest rates and interest rate options in a consistent manner.

Also closely related to our work is the paper by Collin-Dufresne and Goldstein (2001), who find that the yield curve does not span the implied volatility curve of caps and floors. Their main result confirms our finding of independent factors in interest rate options. ${ }^{2}$ They

\footnotetext{
${ }^{1}$ Cointegration, however, is only a special (and extreme) example under which factors are more autocorrelated than residuals. It is thus not a necessary condition for applying the analysis on levels.

${ }^{2}$ Nevertheless, there are a few discrepancies between our results and theirs mainly due to specification differences. Among others, they run regressions on monthly changes without any error correction, thus ignoring the cointegration evidence. Hence, as expected, the explanatory power of their regressions is significantly lower than ours. Another reason for their low explanatory power is that they exclude LIBOR rates and long term (15
} 
compliment our work by focusing on identifying "unspanned" stochastic volatility models that explain the independent movement of the options market. Wadhwa (2000) performs factor analysis on the smoothed daily changes in implied volatility quotes of caplets and swaptions, but does not consider their link to the underlying interest rates.

The paper is structured as follows. The next section describes the data used in the analysis. Section II discusses the estimation techniques and issues for the factor analysis. Section III contains the main result of the paper. Section IV investigates the robustness of the results to variations in data preprocessing and nonlinear transformations. Section V provides some concluding remarks.

\section{Data}

The data set is obtained from Lehman Brothers. It consists of (1) LIBOR rates at maturities of one, two, three, six, and twelve months, (2) swap rates at maturities of two, three, five, seven, ten, 15, and 30 years, and (3) at-the-money swaption Black implied volatilities at option maturities of one, three, six months, and one, two, three, four, five, seven, and ten years. At each of the ten option maturities, we have seven contracts with different underlying swap maturities: one, two, three, four, five, seven, and ten years. All interest rates and interest rate options are on US dollars. The data are daily closing mid quotes from October 30th, 1995 to July 23rd, 2001 (1434 observations).

Swaptions are options to enter a swap contract of a certain tenor at a pre-fixed swap rate (strike). As a standard industry practice, the options contracts (caps, swaptions, etc) are quoted in terms of implied volatilities. Given the quoted volatility, the delivery price is determined based on the current term structure of interest rates and the Black option pricing formula. The industry adoption of the Black model, of course, does not imply an endorsement of the assumptions underlying the Black model, nor does it imply the application of Black

and 30 years) swap rates from their analysis; yet, both Litterman and Scheinkman (1991) and our work indicate that the two ends of the yield curve are the major components of the interest rate curvature factor, which has been found to be highly correlated with the implied volatility surface. 
model for option pricing. Instead, the traders adjust the implied volatility levels across both strikes and maturities to reflect their view on the option price and use the Black formula merely as a nonlinear but monotonic and smooth function to convert the quote into price. A main reason for such a practice is stability concerns.

Table I reports the summary statistics of the data. All series are highly autocorrelated, particularly so for interest rates. Figure 1 plots the term structure of the mean (left panel) and standard deviation (right panel) of the LIBOR and swap rates. The mean yield curve is upward sloping while the standard deviation obtains a maximum value around one-year maturity. Figure 2 depicts the mean (left panel) and standard deviation (right panel) of the swaption implied volatility surface. At short swap maturities, the mean implied volatility exhibits a hump-shaped term structure across the option maturity, a feature also documented in the term structure of implied volatilities of caps and floors, e.g. Backus and Wu (1998). At long swap maturities, the mean term structure decreases monotonically with option maturity. Along the swap maturity dimension, the mean implied volatility is always monotonically declining. The standard deviation of the implied volatility surface declines along both the swap maturity dimension and the option maturity dimension.

In Table II, we report the same summary statistics as in Table I, but on the first difference of the data. A striking feature is that while non-normality in the levels is very moderate, the kurtosis is very large in the first differences, especially for LIBOR rates. This implies that short rates often move discretely on a daily level. The first difference in implied volatilities exhibits larger kurtosis at very short and long maturities than at moderate maturities, potentially an indication of liquidity difference. 


\section{An Approximate Factor Model}

Let $\mathbf{y} \in \mathbb{R}^{N}$ denote the observable interest rates and implied volatilities with $N$ denoting the size of the cross section. Following Chamberlain and Rothschild (1983), we specify an approximate $K$-factor structure:

$$
\mathbf{y}=B_{0}+B \mathbf{x}+\mathbf{e}
$$

where $B_{0} \in \mathbb{R}^{N}$ is a vector of constants, $\mathbf{x} \in \mathbb{R}^{K}$ denotes the $K$ common factors, with $K \leq N$, $B \in \mathbb{R}^{N \times K}$ denotes the factor loading, and $\mathbf{e} \in \mathbb{R}^{N}$ denotes the idiosyncratic risk or pricing error with zero mean $\mathbb{E}[\mathbf{e} \mid \mathbf{x}]=\mathbf{0}$ and covariance matrix $\Sigma_{\mathbf{e}}$. The covariance matrix of the pricing error $\Sigma_{\mathbf{e}}$ is assumed to be a diagonal matrix in a strict factor model but is allowed to have moderate off-diagonal values in an approximate factor model.

Under such a structure, the factors can be identified through standard principal component analysis. We first estimate the covariance matrix of $\mathbf{y}_{t}$,

$$
\widehat{\Sigma}_{\mathbf{y}}=\frac{1}{T} \sum_{t=1}^{T}\left(\mathbf{y}_{t}-\overline{\mathbf{y}}_{t}\right)\left(\mathbf{y}_{t}-\overline{\mathbf{y}}_{t}\right)^{\top}
$$

where $T$ denotes the number of observations for each series, and $\overline{\mathbf{y}}_{t}$ denotes the sample average. Let $D \in \mathbb{R}^{K \times K}$ denotes the diagonal matrix made of the first $K$ largest eigenvalues of the covariance matrix $\Sigma_{\mathbf{y}}$. Let $\mathbf{v} \in \mathbb{R}^{N \times K}$ denotes the corresponding eigenvector matrix. The $K$ factors can then be identified as linear transformations of the observable series:

$$
\mathbf{x}=\mathbf{v}^{\top} \mathbf{y}
$$

The factor loadings, $B$, can then be estimated via linear regression from (1).

The analysis is performed using the following procedure. We first perform principal component analysis on the LIBOR and swap rates to identify the common factors underlying the yield curve. We then investigate how these common factors perform in explaining the variation in the implied volatility surface. Our analysis shows that the yield curve common factors 
only explain slightly over half of the variation in the implied volatility surface. We hence proceed to identify additional factors from the implied volatilities. To do so, we first regress the implied volatilities on the common factors and then identify additional factors from the regression residuals. Due to the inherent indeterminacy problem on the factors, our sequential procedure guarantees that the first three factors in the implied volatility surface coincide with the common factors in the yield curve.

\section{A. Determining the Number of Factors}

To determine the optimal number of factors, we construct a modified version of the test statistic in Connor and Korajczyk (1993). The modification explicitly accounts for the sharp difference in liquidities between the interest rate market and swaptions market.

The liquidity difference shows up most vividly in their difference in bid-ask spreads. The average bid-ask spread for the swap rates is around $0.005 \%$, compared to the mean interest rate level of $6 \%$. In contrast, the average bid-ask spread for swaption implied volatilities is around $0.5 \%$, compared to the mean volatility level of $15 \%$. While the average implied volatility level is less than three times that of the interest rate level, the bid-ask spread is 100 times larger. Thus, applying a universal test statistic to both markets without discretion is likely to overfit the implied volatility surface, or underfit the yield curve. We therefore propose a modified version of the test in Connor and Korajczyk (1993).

The Connor and Korajczyk (1993) test statistic for an approximate $K$-factor model against an $K+1$ factor model is estimated using the following procedure. First, given the factors extracted in (2), we estimate the pricing error $\widehat{e}_{i t}$ of each series via a linear regression. Then we compute the cross-sectional means of the squared residuals at each date, with a simple degree of freedom correction,

$$
\widehat{\mu}_{t}=\frac{\sum_{i=1}^{N} \widehat{e}_{i t}^{2}}{N(1+(K+1) / T-K / N)},
$$

where $N$ denotes the size of the cross section, $K$ denotes the number of factors, and $T$ denotes the length of the time series. Repeat the same procedure to compute $\widehat{\mu}_{t}^{*}$ for the $K+1$ factor 
model. Then calculate the $(T / 2)$-vector $\widehat{\Delta}$ by subtracting $\widehat{\mu}_{t}$ in odd dates from $\widehat{\mu}_{t}^{*}$ in even dates:

$$
\widehat{\Delta}_{s}=\widehat{\mu}_{(2 s-1)}-\widehat{\mu}_{(2 s)}^{*}, \quad s=1, \cdots, T / 2 .
$$

By Theorem 4 in Connor and Korajczyk (1993), under the null hypothesis of $K$ factors, $\widehat{\Delta}$ is asymptotically normally distributed as $N \rightarrow \infty$. One can use the time series of $\widehat{\Delta}$ to calculate its time series mean $\bar{\Delta}$ and standard deviation and test for a positive mean.

Our modified version reflects the rationale that pricing errors within the bid-ask spread of a market should be ignored as they do not represent tradable opportunities. Specifically, we perform a downward adjustment to the cross-sectional means of the estimated squared residuals, $\widehat{\mu}_{t}$ and $\widehat{\mu}_{t}^{*}$, for each market by a maximum of their respective squared bid-ask spreads. The adjusted mean squared residuals are set to zero when negative. In our application, we apply an average bid-ask spread of 0.005 for the interest rate data and 0.5 for the implied volatility series. We use $\widehat{\Delta}_{t}^{A}$ to denote the adjusted version of $\widehat{\Delta}_{t}$.

Intuitively, the unadjusted test of Connor and Korajczyk (1993) argues that an additional factor is needed if the average magnitude of the squared residuals can be reduced significantly by adding such an additional factor. Our adjustment claims that if the average magnitude of the squared residuals is already very small compared to the bid-ask spread of that market, further reducing it is not economically necessary even if the reduction may be statistically significant. The adjustment amounts to a censoring of the average squared pricing error. The asymptotic properties of the test remain unchanged by the adjustment.

\section{B. Exploratory Data Analysis}

We specify the approximate factor model directly on the observed LIBOR and swap rates and implied volatilities. Traditional factor models are mostly specified on (excess) returns of an asset. In our case, to compute returns on the swap and swaption contracts, we need to infer a zero curve or a forward rate curve from the finite number of observations on the term structure, as in Litterman and Scheinkman (1991). Interpolation and smoothing are hence needed. Yet, Björk and Christensen (1999) and Filipović (1999, 2000) show that the commonly used 
smoothing functions, such as the Nelson-Siegel family and cubic spline, are not consistent with any diffusion dynamics. Hence, any such interpolation schemes may induce spurious effects on the factor analysis. Recently, in light of the direct observability of the LIBOR and swap rates, a series of papers have attempted to model the term structure through simply compounded LIBOR or swap rates. Examples include Brace, Gatarek, and Musiela (1997), Glasserman and Kou (2000), Jamshidian (1997), Miltersen, Sandmann, and Sondermann (1997), and Musiela and Rutkowski (1997).

For the same reason, we also apply the factor analysis directly on the implied volatility, rather than on options prices or returns. Furthermore, the movement of the implied volatility surface is relatively separated from the interest rate movement and thus facilitate our identification of independent factors in the options market.

In light of the high persistence in interest rates and implied volatilities (Table I), factor analysis is often performed on first differences instead of levels. We choose to do the opposite. We perform the analysis on levels based on our conjecture that the pricing errors e in (1) are less serially correlated than the factors. The conjecture is based on the well-documented evidence of cointegration among interest rates in, for example, Alexander and Johnson (1994), Bauwens, Deprins, and Vandeuren (1997), Bradley and Lumpkin (1992), Campbell and Shiller (1987), Engle and Granger (1987), Hall, Anderson, and Granger (1992), and Shiller (1981). In our robustness analysis in Section IV, we compare the autocorrelations of the estimated factors and pricing errors and confirm that the pricing errors are significantly less serially correlated than the common factors. Furthermore, such a conjecture also makes better economic sense and implies the existence of a long run equilibrium relationship between the interest rates. Principal component analysis on the levels provides an efficient way of identifying such a long run relationship, e.g. Harris (1997). In Appendix A, we use a simple one-factor example to illustrate that, under our conjecture of reduced persistence in the pricing errors, applying factor analysis to first differences artificially inflates the variance of noise relative to information and thus reduces the efficiency of estimation. The point is further confirmed in the robustness analysis in Section IV, where we repeat our analysis on first differences at different frequencies. 


\section{Results and Discussions}

\section{A. Common Factors in the Interest Rates}

We first perform the factor analysis only on the underlying interest rate market. The analysis is in parallel to that in Litterman and Scheinkman (1991). It provides a benchmark for further analysis of the interest rate options market.

Panel A of Table III reports the test statistics on the optimal number of factors needed for the interest rate market. We also report the percentage of aggregate variation explained by each additional factor. Since the LIBOR and swap rate quotes have very narrow bid-ask spreads, the adjusted and unadjusted test statistics are almost identical. While both a onefactor model and a two-factor model are rejected, the three-factor model cannot be rejected within a $10 \%$ confidence interval. We therefore stop at three factors.

Table IV reports the cumulative variance explained by the first $K$ factors, with $K=1,2,3$. The first factor explains $82.57 \%$ of the aggregate variation. In particular, it explains $97.57 \%$ of the variation in the two-year swap rates, but explains only $58.94 \%$ of the variation in the one month rate and $67.78 \%$ of the variation in the 30 -year swap rate. Incorporating the second factor significantly improves the percentage variation explained at both the short and long ends. The third factor further improves the variance explained at the short and long maturities. Altogether, the three factors explain $99.50 \%$ of the variation in the interest rate market.

Figure 3 depicts the loadings of the three factors on the interest rates. The plots are similar to Figure 2 in Litterman and Scheinkman (1991). The three factors are labeled, respectively, as the level factor (solid line), the slope factor (dashed line), and the curvature factor (dashdotted line). The loading of the first factor is relatively flat across interest rate maturities. The second factor drops the short rate by about 40 basis points but increases the long rate by about 30 basis points. The first two factors combined generate an upward sloping mean yield curve. The curvature factor contributes positively to the middle of the yield curve and negatively to both ends of the curve. It thus enhances the curvature of the yield curve. 


\section{B. Independent Factors in the Derivatives Market}

Once we have extracted the common factors from the interest rate market, we investigate how much variation these common factors explain in the swaptions market. As documented in Panel B of Table III, the three common factors in the interest rate market explain only slightly over half of the variance in the implied volatility surface (59.48\%). Both the adjusted and unadjusted tests highly reject the three common factor model with no additional volatility factors. Obviously, additional factors are needed.

To identify additional factors, we first regress the implied volatility series against the three interest rate factors and then perform principal component analysis on the residuals. Panel B of Table III summarizes the test statistics on the optimal number of additional factors needed for the implied volatilities. As the bid-ask spread is wide for the swaptions market, the adjusted and unadjusted tests produce rather different results. The unadjusted test calls for eight additional factors, while the adjusted test asks only three, based on a $2.5 \%$ confidence level. We hence choose three additional factors for the swaptions market. We label them as volatility factors as they are identified from the implied volatility series.

In Panel $\mathrm{B}$ of Table $\mathrm{V}$, we report the percentage of variance explained by incorporating one additional volatility factor. Compared to the case with only three interest rate factors (Panel A), the explained variance is increased by approximately $25 \%$ to $85.73 \%$. Most of the improvement is at short option expiries across all swap maturities. To illustrate the significance of the improvement, we shade all series which have been explained by more than $90 \%$.

Incorporating two additional factors, as indicated in Panel C, explains $96.11 \%$ of the total variation. With the second volatility factor, most implied volatilities at moderate option and swap maturities can be explained by more than $90 \%$. The deeper shade in the middle region of Panel $\mathrm{C}$ indicates that these series need two volatility factors for their variation to be explained by more than $90 \%$. However, the right bottom corner of the panel is still left unshaded, as the two additional volatility factors are still not enough to improve their explained percentage variation over $90 \%$. In particular, the ten-year option on ten-year swaps is merely explained by $56.79 \%$. 
Incorporating a third factor increases the explained variance to $97.62 \%$, as shown in Panel D of Table V. More importantly, almost all the implied volatility series are explained by more than $90 \%$ (except for three series on ten year swaps). The deepest shade at the right bottom corner of Panel D indicates that the third volatility factor mainly contributes to the long maturity options on long maturity swaps.

\section{Factor Loadings on the Implied Volatility Surface}

Figure 4 plots the loading of the three common factors on the implied volatility surface. Overall, the interest rate level factor (top panel) contributes negatively to the implied volatility surface. The impact is largest at $-1.26 \%$ on one month options, but smallest at $-0.18 \%$ on ten year options, both on one year swaps.

The impact of the interest rate slope factor (middle panel) is mostly positive. The largest impact is again on one month options on one year swaps at $1.70 \%$, but its loadings on long term options on long maturity swaps are close to zero.

Of the three interest rate factors, the largest impact on implied volatility comes from the curvature factor, as depicted in the bottom panel of Figure 4. While the impacts of the level and slope factors are all less than two percent, the impact of the curvature factor can be as high as ten percent. A positive shock to the curvature factor generates a large negative shock to the implied volatility level, especially at the short option and swap maturities.

Figure 5 graphs the loading of the additional volatility factors on the implied volatility surface. The loading of the first volatility factor (top panel) is about $0.2 \%$ at short option maturities, but is close to zero at long option maturities. Its impact along the swap maturity dimension is relatively flat.

The second volatility factor (middle panel) contributes positively to the implied volatility surface at short option maturities, but negatively at moderate to long option maturities. As a result, the second volatility factor generates a downward slope in the term structure of the 
implied volatility surface along the option maturity dimension. The term spread generated from this factor is about $0.2 \%$ to $0.35 \%$, depending on the underlying swap maturities.

The third volatility factor (bottom panel) contributes positively at the two ends of the option maturities, but negatively in the middle. The largest absolute impact is on very long option maturities (ten year) at around $0.3 \%$.

The distinct actions of the three volatility factors are mostly along the option maturity dimension, their impact along the swap maturity dimension is relatively flat. Analogous to the impacts of the three interest rate factors on the yield curve, the three volatility factors can also be regarded as capturing, respectively, the level, the slope, and the curvature of the implied volatility term structure along the option maturity dimension.

\section{Mimicking Portfolios}

An advantage of the principal component analysis is that one can directly extract the factors as a linear combination of the observables. Having portfolios that mimic a particular factor allows us to investigate further the properties of the unobservable factors and the impact of these factors on other fixed income securities.

Table VI reports the correlations between the interest rates and the mimicking portfolios of the six factors: the three interest rate factors and the three additional volatility factors. The interest rate level factor is positively correlated with all interest rates, with an almost perfect correlation (0.99) with the two-year swap rate. Hence, as evidenced before, the level factor can be well proxyed by the two-year swap rate.

The mimicking portfolio for the slope factor is negatively correlated with short rates but positively correlated with long rates and thus generates non-parallel movement in the yield curve. While the eigenvector provides a direct way of forming the mimicking portfolio, we can approximate the second factor with a simpler and more intuitive portfolio. In particular, if we form a spread portfolio by going long one unit of the 30-year swap rate and shorting one unit of the one-month LIBOR rate, the spread portfolio has an almost perfect correlation (0.98) 
with the slope factor, thus confirming that the second factor indeed captures the slope of the curve.

The mimicking portfolio for the curvature factor is positively correlated with moderate maturity swaps but negatively correlated with both short and long term interest rates. We construct a portfolio which goes long 1.6 units of the two-year swap rate and shorting one unit of the one-month LIBOR rate and 1.5 units of the 30-year swap rate. Such a three instrument portfolio is commonly referred to as a butterfly, or a barbell. It captures the curvature of the yield curve. In the last row of Table VI, we report the correlation of this butterfly portfolio with the the six factors. Not surprisingly, its correlation with the third factor (the curvature factor) is nearly perfect (0.99). In the language of fixed income risk management, the three common factors are referred to as the duration, curve, and butterfly risk, respectively. Duration hedging essentially focuses on the risk of the level factor where interest rates are assumed to move in parallel. Yet, the curve can flatten or steepen over time, thus generating slope changes. Furthermore, the curvature of the yield curve also changes and its movement can be captured by that of a butterfly portfolio.

The last three columns of Table VI report the correlations of each interest rate series with the three volatility factors. The correlations are all very close to zero, implying that the volatility factors are not only independent of the three interest rate factors by construction, but also uncorrelated with the underlying yield curve altogether. This is the most intriguing part of the result. In theory, in an efficient market, any factors in interest rate options should also show up in the yield curve. Therefore, the "independent" volatility factors may imply potential inefficiency in the options market.

Table VII reports the correlations of each implied volatility series with the mimicking portfolios of the six factors. The correlations between the interest rate level factor and the implied volatilities are all negative, ranging from -0.24 to -0.60 , with an average of -0.48 . These negative correlations point to a direction where the Black model is misspecified. In the Black model, the diffusion of the swap rate is assumed to be proportional to the swap rate level. Thus, the negative correlation between the Black volatility and the interest rate level 
factor may imply that the proportionality assumption is overstating the dependence of the diffusion on the interest rate level. ${ }^{3}$

Again, we use shades to highlight the relative contribution of each factor. In particular, in Table VII, we shade all the series whose correlation with the specific factor is higher than 0.5 in absolute value. The shading of the top right triangle in Panel A illustrates that the interest rate level factor is more correlated with options on long swap maturities than with options on short swap maturities.

The correlations of the implied volatilities with the interest rate slope factor (Panel B) are all positive, ranging from zero at long swap and option maturities to 0.31 at short swap and option maturities. The correlations are mostly small and all below 0.5. Nevertheless, in contrast to the level factor, the slope factor is more correlated with implied volatilities on short swap maturities.

Similar to the interest rate level factor, the interest rate curvature factor (Panel C) also possesses high correlations with the implied volatilities. Unlike the level factor, however, the curvature factor is more correlated with options on short maturity swaps. Litterman and Scheinkman (1991) claim that the curvature factor is closely related to the volatility level of the interest rates. Our finding confirms such a claim and further indicates that the curvature factor is more correlated with the volatility level of short term interest rates. On the other hand, the implied volatilities of options on long term swaps are more correlated with the interest rate level factor.

Panel D of Table VII reports the correlations of the first volatility factor with the implied volatility surface. The correlations vary greatly across option maturities, but are relatively flat across swap maturities. It has large positive correlation with implied volatilities of short term options, but its correlation with long term option implied volatilities is close to zero. Such a factor complements the interest rate level and curvature factors in further explaining the level of the volatilities. We hence label it as the volatility level factor.

\footnotetext{
${ }^{3}$ For example, a square root dependence would generate a negative correlation.
} 
Panel E of Table VII reports the correlation between the second volatility factor and the implied volatility surface. The correlations are positive with short term options but negative with long term options. The correlations across the swap dimension are again relatively flat. Hence, the factor contributes to the slope of the implied volatility term structure along the option expiry dimension. To illustrate this idea, we form a series of "spread" portfolios by going long one unit of the one-month volatility and shorting four units of the five-year volatility for each underlying swap rate. In the last row of Panel E, we report the correlations of the second volatility factor with these seven spread portfolios. While not perfect, the correlations of the second volatility factor with these spread portfolios are very high, confirming the contribution of this factor to the slope of the option term structure. We thus label the second volatility factor as the volatility spread factor. Furthermore, while the volatility level factor is more correlated with short term options, the slope factor is more correlated with moderate to long term options.

The correlations of the third volatility factor with the implied volatilities are summarized in Panel F of Table VII. Overall, the correlations are very small except at very long option and swap maturities. The correlations are positive at very short and very long option maturities, but negative in the middle, forming a "butterfly" structure. We exploit this structure and construct a series of butterfly portfolios corresponding to each underlying swap rate by going long one unit of the one-month volatility and two units of ten-year volatility and shorting two units of the seven-year volatility. The last row of Panel F reports the correlation between the third factor and these portfolios. The correlation is around 0.9 for all swap maturities. We hence label the third volatility factor as the volatility butterfly factor.

The "spread" and "butterfly" portfolios are formed only for illustrative purposes. They serve as motivations for our christening of the three volatility factors. Thus, we make no attempt in optimizing the exact formulation. Furthermore, we construct a series of spread and butterfly portfolios corresponding to each underlying swap maturity to illustrate the idea that the volatility factors are mainly capturing the term structure along the option maturity dimension, but are relatively insensitive to the choice of the underlying swap maturity. In practice, obviously, we do not need to form the spread and butterfly portfolios within the same 
swap maturity, nor do we need to form so many portfolios of each type. One portfolio for each factor is enough.

In short, the three volatility factors capture, respectively, the level, slope, and curvature of the implied volatility term structure along the option maturity dimension. Their contributions along the swap maturity dimension, however, are relatively flat, implying that these factors are mainly capturing the time-aggregation and conditioning of the volatility process, which determines the term structure of the volatility along the option maturity dimension. The volatility term structure along the swap maturity dimension, on the other hand, is captured by the interest rate factors. In particular, the interest rate level factor is more correlated with implied volatilities on long term swaps while the interest rate curvature factor contributes more to implied volatilities on short term swaps. While smaller in magnitude, the interest rate slope factor is also more correlated with implied volatilities on short maturity swaps.

\section{Robustness Analysis}

In this section, we discuss the robustness of our findings. Specifically, we consider the effects of data choice, differencing, and nonlinearity on our results.

\section{A. Data Choice}

The choices of swap rates and swaptions of the specific maturities are determined partly by the availability of the respective contracts and quotes and partly by the real information content of each contract. For example, swap contracts with 15 and 30 year maturities are available at a later date than shorter term swaps. Including them in the sample results in a shorter sample period. Nevertheless, we choose to leave them in our sample because we find that they are the key components of the third yield curve factor. On the other hand, we decide to exclude swaptions on very long swaps (with maturity higher than ten years) from our sample. These option contracts are available at a much later date than the other series. Including them in the sample would imply a significant truncation of the sample length. We are also concerned with 
the liquidity of the market on these very long swap options. Most importantly, however, we have found that the volatility factors mainly capture the variation along the option maturity dimension. Thus, incorporating additional swaptions with different underlying swap contracts does not significantly increase the information content of the sample.

Also available are implied volatility quotes on interest rate caps, which are options on threemonth LIBOR rates. We also exclude them from our sample for several reasons. First, we find that the information content in interest rate caps are sufficiently captured in the swaptions implied volatilities. We have repeated our exercises with cap implied volatility only, and then with cap and swaption implied volatility combined. In both cases, we find that independent factors exist in the options market. The factor loadings also possess similar shapes, indicating that we capture roughly the same factors from caps and swaptions. Again, as we have found from previous analysis, the volatility factors mainly capture the term structure variations along the option maturity dimension. Implied volatilities on caps and on swaptions can both capture this dimension independently. Nevertheless, as the cross section is much larger with swaptions, the identification of the volatility factors becomes more stable and robust. More importantly, with swaptions, we can better distinguish the contributions of the interest rate factors and the volatility factors. Including caps in our sample does not vary our conclusions, but it increases the burden of exposition. Finally, while a swaption can be regarded as an option on a portfolio of forward rates, a cap is a portfolio of options (caplets) at different maturities on one single LIBOR rate. As such, out of the whole portfolio of options for each cap contract, only one caplet can be truly at-the-money. The implied volatility quote of a cap is hence an average number which is more likely to be contaminated by the potential skewness of the interest rate distribution.

\section{B. Cointegration and Differencing}

In our principal component analysis, the choice of levels versus differences hinges crucially on the conjecture that the pricing errors are less serially correlated than the common factors. See Appendix A for details. To confirm that our conjecture is correct, we perform the following analysis. Under our approximate six factor model, we extract both the common factors and the 
idiosyncratic pricing errors for each series. We then compare their persistence. The first-order autocorrelations of the six factors are, respectively, 0.9957, 0.9920, 0.9877, 0.9711, 0.9888, and 0.9824, while the average first-order autocorrelation for the pricing errors is only 0.9098 , much smaller than any of the six factors. More formally, we decompose the time series into two parts,

$$
\mathbf{y}=\widehat{\mathbf{y}}+\widehat{\mathbf{e}}
$$

where $\widehat{\mathbf{y}}=B_{0}+B \mathbf{x}$ denotes the explained part of the series and $\widehat{\mathbf{e}}$ denotes the model-implied pricing error. We then test whether the first-order autocorrelation of $\widehat{\mathbf{y}}$, denoted by $\rho_{\widehat{y}}$, is significantly greater than the first-order autocorrelation of $\widehat{\mathbf{e}}$, denoted by $\rho_{\widehat{e}}$. In particular, applying the standard $Z$-test notation for a correlation coefficient,

$$
Z_{1}=\frac{1}{2} \ln \frac{1+\rho_{\widehat{y}}}{1-\rho_{\widehat{y}}}, \quad Z_{2}=\frac{1}{2} \ln \frac{1+\rho_{\widehat{e}}}{1-\rho_{\widehat{e}}}
$$

we construct a test statistic for the null hypothesis that the two correlation coefficients are the same,

$$
Z=\frac{Z_{1}-Z_{2}}{\sigma}
$$

where $\sigma=\sqrt{2 /(T-3)}$ and $T$ is the number of observations. Under the null hypothesis, $Z$ is normally distributed with zero mean and unit variance. Table VIII reports the sample estimates for the test for each series. The statistics are significantly positive for all series, thus confirming our conjecture that the errors are less persistent than the factors. Our choice of levels instead of differences are therefore well founded. The test results are also in line with the cointegration evidence in the literature.

To investigate how the results would be distorted by taking differences, we repeat our principal component analysis based on daily, weekly, and monthly differences. The daily difference is straightforward as first differences of the daily data. Weekly difference is based on Wednesdays. Monthly difference is based on every four weeks. In five weeks when Wednesday is a holiday, we use Tuesday instead. We have 299 weekly observations. 
We summarize the results on first differences in Table IX. ${ }^{4}$ The first three panels correspond to the three frequencies: daily, weekly, and monthly first differences. For comparison, we also report the summary result on levels in the bottom panel. In the first row of each panel, we report the percentage of aggregate variance explained by the first $K$ factors. Parallel to the analysis on levels, we report the explained variance for interest rates for the first three factors and then the explained variance for implied volatilities for each additional volatility factor. The case of zero additional volatility factor represents the case where we explain the implied volatility surface with only interest rate factors.

An obvious impact of taking first differences is that the percentage of variance explained is significantly smaller than in the levels case. For example, while the three factors extracted from interest rate levels can explain $59.48 \%$ of the variation in the implied volatility surface, the three factors extracted from interest rate daily differences can only explain $3 \%$ of the variance. The percentage increases to $8 \%$ for weekly differences, and $18 \%$ for monthly differences, all significantly lower than those explained by factors from interest rate levels. This confirms our argument in Appendix A that, when the pricing error is less persistent than the common factors, taking differences reduces the relative significance of information versus noise.

The reduced significance of information versus noise also reduces the power of the test for optimal number of factors. For example, in the case of daily differences, we cannot reject a one-factor interest rate model although it only explains $72.38 \%$ of the interest rate variation. Even more strikingly, although the three interest rate factors only explain a meager $3 \%$ of the variation in implied volatility surface, the adjusted test does not identify the need for any additional volatility factors! The null hypothesis of zero additional volatility factor cannot be rejected with a $p$-value of 0.11 . Thus, based on the daily difference, one would conclude that the interest rate factors cannot explain the variations in the implied volatilities at all, but most of these variations are idiosyncratic noise. The real reason, however, is that by taking daily differences, one artificially inflates the noise relative to the informational signal to such an extent that it is no longer possible to effectively identify the common movements in the

\footnotetext{
${ }^{4}$ More detailed analysis based on first differences, corresponding to each of the tables and figures in this paper, are available upon request.
} 
implied volatility surface. This partially explains the low explanatory power of the regressions in Collin-Dufresne and Goldstein (2001), who regress monthly changes in the prices of straddles against the monthly changes in swap rates.

\section{Impacts of Nonlinear Transformations}

Our analysis assumes an approximate linear structure between the common factors and the observable series on interest rates and implied volatilities. Most term structure models specify the factors in terms of the continuously compounded zero rate or forward rate. The simply compounded LIBOR rates and swap rates are nonlinear transformations of these zero rates. Therefore, even if the factors are linear in the zero or forward rates, as in the affine class of Duffie and Kan (1996), they are likely to be nonlinear in terms of the LIBOR and swap rates. Under the quadratic class of Leippold and Wu (2001), even the continuously compounded spot or forward rates are not linear in factors. Furthermore, due to the nonlinear relations between swap rates and swaptions, the implied volatilities are necessarily nonlinear in the same factors. Our linear specification at best represents an approximation.

To investigate the effects of potential nonlinearity on our principal component analysis, we consider the following perturbation strategy. We create an artificially large cross section out of the interest rate data by including powers (both positive and negative) of the interest rates in the sample. The underlying idea is simple. If the factors are indeed nonlinear in the interest rates, we should be able to approximately capture such relations through a Laurent power series expansion. We then apply principal component analysis on the expanded sample and investigate the performance of factor models with different number of factors. Table $\mathrm{X}$ summarizes the result of the exercise. As we expand the interest rates by its powers up to the sixth order, the percentages of variation explained by the first three factors are above $99.5 \%$ for all cases. Instead of experiencing a decline in the percentage as we expand the series further, the percentage actually experience a slight increase. Only when we incorporate the implied volatility series, do we discern a significant drop in percentage and the need for more factors. The exercise confirms that the independent factors identified from the options market are not 
a spurious result of our linear approximation. They may represent dynamics that are absent from the yield curve.

\section{Concluding Remarks}

Inspired by the sharply different behaviors of interest rate traders and option players, we try to clarify whether the same finite dimensional dynamic system spans both the yield curve and the implied volatility surface of interest rate options. The answer is negative. The options market exhibits factors independent of the underlying yield curve. The finding has important implications for pricing and hedging in the fixed income market, particularly so for securities spanning both markets, such as callable/puttable bonds and mortgage backed securities.

On top of our research agenda is to investigate the structural nature of these independent volatility factors. One conjecture is that the swaptions market may be inefficient. Compared to the underlying swap market, the options market is much smaller and is dominated by a few large players. The independent volatility factors may represent the position adjustment of these large players. To confirm such a conjecture, one needs to identify the historic positions of these large players and investigate their correlations with the volatility factors.

Another conjecture is related to the finite dimensional representation of the yield curve. If there does not exist a finite or low dimensional representation, hedging options would need a large number of interest rate instruments. In that case, even small transaction cost on each instrument implies very significant total cost for daily adjustment of such a hedging portfolio. Thus, in practice, complete hedging of option positions becomes infeasible. Option prices may therefore exhibit independent movements which are not spanned by the yield curve. Under the framework of Heath, Jarrow, and Morton (1992), Björk and Gombani (1999) and Björk and Svensson (2000) provide the necessary and sufficient conditions for the existence of a finite dimensional Markovian state space realization. Under these conditions, the finite number of factors can all be explained by a minimum set of benchmark forward rates. Our statistical evidence for independent volatility factors calls for further theoretical efforts in answering 
the following question: Does there exist a finite dimensional representation compatible with independent volatility factors, and if so, what are the necessary and sufficient conditions?

Since we find that three factors capture $99.5 \%$ of the variation on the yield curve, an infinite dimensional representation seems unlikely, unless there exists an identification problem. Potentially, some factors may have statistically negligible impacts on the yield curve but significant impacts on the implied volatility surface. In this case, the "independent" volatility factors are not truly independent of the yield curve, but the dependence exhibits no statistical significance. The task is then to identify a model structure which exhibits such a feature. In future research for model design, one should fully exploit the $m+n$ structure identified in this paper, where $m$ denotes the number of common factors identified from the yield curve and $n$ denotes the number of independent factors from the implied volatility surface. Given such a structure, one can calibrate the $m$ common factors to the yield curve, pretending that the $n$ independent volatility factors do not exist, and then calibrate the $n$ factors to the implied volatility surface. Such a separation would achieve significant efficiency gains.

Finally, Collin-Dufresne and Goldstein (2001) propose a class of "unspanned" stochastic volatility (USV) models to account for the independent factors in the implied volatility surface. They further claim that all stochastic volatility HJM models exhibit USV. A future line of research is to empirically investigate and verify the performance of these USV models in generating the $3+3$ factor structures identified in this paper. 


\section{Appendix A. Levels versus Differences}

We use a simple one-factor example to illustrate that taking differences reduces the relative significance of information versus noise when the pricing error is less serially correlated than the common factor.

Consider a simple one-factor model,

$$
y_{t}=b_{0}+b x_{t}+e_{t}
$$

where both the common factor $x_{t}$ and the pricing error $e_{t}$ follow an $\operatorname{AR}(1)$ process:

$$
\begin{aligned}
& x_{t}=\rho_{x} x_{t-1}+\gamma_{t} \\
& e_{t}=\rho_{e} e_{t-1}+\varepsilon_{t}
\end{aligned}
$$

with $\gamma$ and $\varepsilon$ being iid white noises. The relative significance of information versus noise is captured by the signal to noise ratio, $\xi$, defined as the ratio of the variance of the explanatory variable to the variance of the residual.

When applying the analysis on the levels, the signal to noise ratio is given by,

$$
\xi_{\text {level }}=\frac{\sigma_{x}^{2}}{\sigma_{e}^{2}}
$$

When we apply the analysis to the first differences, the signal to noise ratio becomes

$$
\xi_{\text {difference }}=\frac{2\left(1-\rho_{x}\right) \sigma_{x}^{2}}{2\left(1-\rho_{e}\right) \sigma_{e}^{2}}=\frac{1-\rho_{x}}{1-\rho_{e}} \xi_{\text {level }} .
$$

Thus, when the pricing error is more persistent than the common factor $\left(\rho_{e}>\rho_{x}\right)$, taking first differences increases the signal to noise ratio and increases the efficiency of the estimation. However, if the common factor is more persistent than the pricing error, the opposite will be true. Taking differences reduces the efficiency.

Therefore, the decision on whether the analysis should be performed on the level or first difference hinges crucially on the relative persistence of the common factor versus that of the pricing error. The example can readily be extended to a multifactor setting if we replace the single factor $x_{t}$ by the explained portion of the series $\hat{y}_{t}$ in the definition of the signal to noise ratio. Then, the decision depends upon the relative persistence of $\hat{y}=B_{0}+B \mathbf{x}$, which is a portfolio of the common factors, versus that of the pricing error. 


\section{References}

Alexander, Carol, and Andrew Johnson, 1994, Dynamic links, RISK 7, 56-61.

Backus, David, and Liuren Wu, 1998, The "hump-shaped" mean term structure of interest rate derivative vols, manuscript, Fordham University.

Bauwens, Luc, Dominique Deprins, and Jean-Pierre Vandeuren, 1997, Modeling interest rates with a cointegrated VAR-GARCH model, manuscript, Université Catholique de Louvain.

Björk, Tomas, and Bent Jesper Christensen, 1999, Interest rate dynamics and consistent forward rate curves, Mathematical Finance 9, 323-348.

Björk, Tomas, and Andrea Gombani, 1999, Minimal realizations of forward rates, Finance and Stochastics $3,413-432$.

Björk, Tomas, and Lars Svensson, 2000, On the existence of finite dimensional realization for nonlinear forward rate models, Mathematical Finance forthcoming.

Brace, A., D. Gatarek, and Marek Musiela, 1997, The market model of interest rate dynamics, Mathematical Finance 7, 127-255.

Bradley, Michael G., and Stephen A. Lumpkin, 1992, The treasury yiled curve as a cointegrated system, Journal of Financial and Quantitative Analysis 27, 449-463.

Campbell, John, and Robert Shiller, 1987, Cointegration and tests of present value models, Journal of Political Economy 95, 1062-1088.

Chamberlain, Gary, and Michael Rothschild, 1983, Arbitrage, factor structure and mean-variance analysis on large asset markets, Econometrica 51, 1281-1304.

Collin-Dufresne, Pierre, and Robert S. Goldstein, 2001, Do bonds span the fixed income markets? theory and evidence for unspanned stochastic volatility, manuscript, Carnegie Mellon University Pittsburgh.

Connor, Gregory, and Robert A. Korajczyk, 1993, A test for the number of factors in an approximate factor model, Journal of Finance 48, 1263-1291.

Duffee, Gregory R., 1996, Idiosyncratic variation of treasury bill yields, Journal of Finance 51, 527-551.

Duffie, Darrell, and Rui Kan, 1996, A yield-factor model of interest rates, Mathematical Finance 6, 379-406.

Engle, Robert F., and C. W. J. Granger, 1987, Co-integration and error correction: Representation, estimation, and testing, Econometrica 55, 251-276. 
Filipović, Damir, 1999, A note on the nelson-siegel family, Mathematical Finance 9, 349-359.

Filipović, Damir, 2000, Exponential-polynomial families and the term structure of interest rates, Bernoulli 6, 1-27.

Glasserman, Paul, and Steven G. Kou, 2000, The term structure of simple forward rates with jump risk, manuscript, Columbia University New York.

Hall, Anthony D., Heather M. Anderson, and Clive W.J. Granger, 1992, A cointegration analysis of treasury bill yields, Review of Economics and Statistics 71, 116-126.

Harris, David, 1997, Principal components analysis of cointegrated time series, Econometric Theory 13, $529-557$.

He, Hua, 2000, Modeling term structures of swap contracts, Working paper 00-16 Yale School of Management New Haven.

Heath, David, Robert Jarrow, and Andrew Morton, 1992, Bond pricing and the term structure of interest rates: A new technology for contingent claims valuation, Econometrica 60, 77-105.

Jamshidian, F., 1997, Libor and swap market models and measures, Finance and Stochastics 1, $293-330$.

Knez, Peter J., Robert Litterman, and Jose Scheinkman, 1994, Explorations into factors explaining money market returns, Journal of Finance 49, 1861-1882.

Leippold, Markus, and Liuren Wu, 2001, Asset pricing under the quadratic class, Journal of Financial and Quantitative Analysis forthcoming.

Litterman, Robert, and Jose Scheinkman, 1991, Common factors affecting bond returns, Journal of Fixed Income 1, 54-61.

Liu, Jun, Francis A. Longstaff, and Ravit E. Mandell, 2000, The market price of credit risk: An empirical analysis of interest rate swap spreads, manuscript, UCLA.

Longstaff, Francis A., 2000, The term structure of very short-term rates: New evidence for the expectation hypothesis, Journal of Financial Economics 58, 397-415.

Masson, Valery, 2000, Swap spreads, what has changed?, Euro Derivatives-Risk pp. 4-5.

Miltersen, Kristian R., Klaus Sandmann, and Dieter Sondermann, 1997, Closed-form solutions for term structure derivatives with lognormal interest rates, Journal of Finance 52, 409-430.

Musiela, Marek, and Marek Rutkowski, 1997, Continuous-time term structure models: Forward measure approach, Finance and Stochastics 1, 261-291. 
Shiller, Robert J., 1981, Do stock prices move too much to be justified by subsequent changes in dividends?, American Economic Review 71, 421-36.

Wadhwa, Pavan, 2000, An empirical analysis of the common factors governing us dollar-libor implied volatility movements, Working paper, J. P. Morgan New Yok. 
Table I

\section{Summary Statistics}

Entries are summary statistics of the interest rate and implied volatility data used in the factor analysis. Mean, Std, Skewness, Kurtosis, and Auto denote, respectively, the sample estimates of the mean, standard deviation, skewness, kurtosis, and first-order autocorrelation. In the maturity column, $\mathrm{m}$ denotes months and y denotes years. To save space, we only report the average statistics for swaptions at each option maturity. The data are daily closing mid quotes from Lehman Brothers, ranging from October 30th, 1995 to July 23rd, 2001 (1434 observations).

\begin{tabular}{|c|c|c|c|c|c|}
\hline Maturity & Mean & Std & Skewness & Kurtosis & Auto \\
\hline \multicolumn{6}{|c|}{ LIBOR and Swap Rates } \\
\hline $1 \mathrm{~m}$ & 5.582 & 0.568 & -0.293 & 1.422 & 0.993 \\
\hline $2 \mathrm{~m}$ & 5.612 & 0.579 & -0.406 & 1.575 & 0.994 \\
\hline $3 \mathrm{~m}$ & 5.643 & 0.600 & -0.444 & 1.504 & 0.995 \\
\hline $6 \mathrm{~m}$ & 5.701 & 0.639 & -0.452 & 1.184 & 0.995 \\
\hline $1 \mathrm{y}$ & 5.847 & 0.691 & -0.268 & 0.393 & 0.995 \\
\hline $2 y$ & 6.015 & 0.681 & -0.089 & -0.395 & 0.995 \\
\hline $3 y$ & 6.144 & 0.650 & -0.016 & -0.635 & 0.995 \\
\hline $5 \mathrm{y}$ & 6.307 & 0.613 & -0.013 & -0.771 & 0.995 \\
\hline $7 \mathrm{y}$ & 6.420 & 0.593 & -0.057 & -0.831 & 0.995 \\
\hline $10 \mathrm{y}$ & 6.547 & 0.572 & -0.105 & -0.905 & 0.994 \\
\hline $15 \mathrm{y}$ & 6.684 & 0.543 & -0.136 & -1.010 & 0.994 \\
\hline $30 \mathrm{y}$ & 6.750 & 0.504 & -0.176 & -1.089 & 0.995 \\
\hline \multicolumn{6}{|c|}{ Swaption Implied Volatility } \\
\hline $1 \mathrm{~m}$ & 15.720 & 3.557 & 0.702 & 0.222 & 0.989 \\
\hline $3 \mathrm{~m}$ & 15.884 & 3.227 & 0.682 & 0.283 & 0.991 \\
\hline $6 \mathrm{~m}$ & 16.081 & 2.795 & 0.493 & -0.248 & 0.991 \\
\hline $1 \mathrm{y}$ & 16.313 & 2.301 & 0.246 & -0.870 & 0.992 \\
\hline $2 y$ & 16.280 & 1.831 & 0.197 & -0.679 & 0.991 \\
\hline $3 y$ & 15.942 & 1.645 & 0.163 & -0.585 & 0.990 \\
\hline $4 \mathrm{y}$ & 15.555 & 1.487 & 0.138 & -0.497 & 0.989 \\
\hline $5 \mathrm{y}$ & 15.139 & 1.349 & 0.114 & -0.458 & 0.988 \\
\hline $7 \mathrm{y}$ & 14.007 & 1.272 & 0.220 & -0.281 & 0.989 \\
\hline $10 \mathrm{y}$ & 12.531 & 1.330 & 0.253 & -0.260 & 0.989 \\
\hline
\end{tabular}


Table II

\section{Summary Statistics of the First Difference}

Entries are summary statistics on the first difference of the interest rates and implied volatilities. Mean, Std, Skewness, Kurtosis, and Auto denote, respectively, the sample estimates of the mean, standard deviation, skewness, kurtosis, and first-order autocorrelation. In the maturity column, m denotes months and y denotes years. To save space, we only report the average statistics for swaptions at each option maturity. The data are daily closing mid quotes from Lehman Brothers, ranging from October 30th, 1995 to July 23rd, 2001 (1434 observations).

\begin{tabular}{|c|c|c|c|c|c|}
\hline Maturity & Mean & Std & Skewness & Kurtosis & Auto \\
\hline \multicolumn{6}{|c|}{ LIBOR and Swap Rates } \\
\hline $1 \mathrm{~m}$ & -0.001 & 0.047 & 0.662 & 147.975 & 0.040 \\
\hline $2 \mathrm{~m}$ & -0.002 & 0.037 & 4.961 & 227.568 & 0.073 \\
\hline $3 \mathrm{~m}$ & -0.002 & 0.033 & 0.082 & 106.412 & 0.124 \\
\hline $6 \mathrm{~m}$ & -0.002 & 0.033 & -2.533 & 30.588 & 0.138 \\
\hline $1 \mathrm{y}$ & -0.001 & 0.042 & -0.496 & 12.052 & 0.138 \\
\hline $2 \mathrm{y}$ & -0.001 & 0.054 & 0.508 & 6.742 & 0.123 \\
\hline $3 y$ & -0.001 & 0.057 & 0.477 & 5.529 & 0.122 \\
\hline $5 y$ & -0.000 & 0.058 & 0.324 & 3.302 & 0.105 \\
\hline $7 \mathrm{y}$ & -0.000 & 0.058 & 0.351 & 2.924 & 0.100 \\
\hline $10 \mathrm{y}$ & -0.000 & 0.058 & 0.406 & 2.589 & 0.104 \\
\hline $15 \mathrm{y}$ & -0.000 & 0.056 & 0.427 & 2.612 & 0.096 \\
\hline $30 \mathrm{y}$ & -0.000 & 0.050 & 0.288 & 1.670 & 0.060 \\
\hline \multicolumn{6}{|c|}{ Swaption Implied Volatility } \\
\hline $1 \mathrm{~m}$ & 0.001 & 0.510 & 0.720 & 12.123 & 0.093 \\
\hline $3 \mathrm{~m}$ & 0.001 & 0.413 & 0.871 & 15.701 & 0.135 \\
\hline $6 \mathrm{~m}$ & 0.001 & 0.349 & 0.238 & 21.987 & 0.119 \\
\hline $1 \mathrm{y}$ & -0.000 & 0.267 & 0.815 & 9.447 & 0.190 \\
\hline $2 \mathrm{y}$ & -0.001 & 0.230 & 0.545 & 7.043 & 0.156 \\
\hline $3 \mathrm{y}$ & -0.000 & 0.213 & 0.504 & 7.572 & 0.155 \\
\hline $4 \mathrm{y}$ & -0.000 & 0.201 & 0.335 & 8.102 & 0.135 \\
\hline $5 \mathrm{y}$ & 0.000 & 0.194 & 0.129 & 8.579 & 0.126 \\
\hline $7 \mathrm{y}$ & 0.000 & 0.179 & -0.069 & 13.163 & 0.072 \\
\hline $10 \mathrm{y}$ & -0.001 & 0.182 & 0.248 & 27.260 & 0.027 \\
\hline
\end{tabular}


Table III

Tests for Approximate $K$ Factor Models

The first column, $K$, denotes number of factors. The second column, "Variance", denotes the percentage of aggregate variance explained by $K$ factors. $\bar{\Delta}$ denotes the time series mean of the test statistic for $K$ factors versus $K+1$ factors. $\bar{\Delta}^{A}$ is the time series mean of the adjusted test accounting for transaction costs. The " $t$-Value" columns (fourth and seventh) divide column three and six, respectively, by its time series estimated standard error (scaled by the square root of the time series sample size) to form a $t$-statistic. The fifth and eight " $p$-Value" columns report the one-sided tail area of the $t$-statistic found in the third and sixth columns, respectively. The bolded numbers reflect the minimum number of factors under which the model cannot be rejected under $2.5 \%$ confidence interval, based on each statistic. Panel A reports variance explained and tests on the interest rate market. Panel B reports variance explained and tests on additional factors needed for explaining the implied volatility surface. $K=0$ denotes the case where the implied volatility surface is explained by the three interest rate factors with zero additional volatility factors.

\begin{tabular}{|c|c|c|c|c|c|c|c|}
\hline \multirow[t]{2}{*}{$K$} & \multirow{2}{*}{$\begin{array}{c}\text { Variance } \\
\%\end{array}$} & \multicolumn{3}{|c|}{ Unadjusted Tests } & \multicolumn{3}{|c|}{ Adjusted Tests } \\
\hline & & $\bar{\Delta}$ & $t$-Value & $p$-Value & $\bar{\Delta}^{A}$ & $t$-Value & $p$-Value \\
\hline \multicolumn{8}{|c|}{ A. Factors in Interest Rates } \\
\hline 1 & 82.57 & 0.06 & 2.74 & 0.00 & 0.06 & 2.74 & 0.00 \\
\hline 2 & 98.28 & 0.01 & 2.80 & 0.00 & 0.01 & 2.80 & 0.00 \\
\hline 3 & 99.50 & 0.00 & 1.31 & 0.10 & 0.00 & 1.31 & 0.10 \\
\hline
\end{tabular}

B. Additional Factors in Implied Volatilities

\begin{tabular}{llllllll}
\hline 0 & 59.48 & 1.33 & 5.06 & 0.00 & 1.30 & 4.96 & 0.00 \\
1 & 85.73 & 0.53 & 4.83 & 0.00 & 0.48 & 4.30 & 0.00 \\
2 & 96.11 & 0.08 & 6.27 & 0.00 & 0.03 & 2.79 & 0.00 \\
3 & 97.62 & 0.06 & 5.37 & 0.00 & 0.01 & $\mathbf{1 . 8 7}$ & $\mathbf{0 . 0 3}$ \\
4 & 98.65 & 0.03 & 4.48 & 0.00 & 0.00 & 0.72 & 0.24 \\
5 & 99.15 & 0.01 & 3.42 & 0.00 & 0.00 & 0.62 & 0.27 \\
6 & 99.39 & 0.01 & 3.13 & 0.00 & 0.00 & 0.51 & 0.30 \\
7 & 99.53 & 0.00 & 2.08 & 0.02 & 0.00 & 0.31 & 0.38 \\
8 & 99.59 & 0.00 & $\mathbf{1 . 8 7}$ & $\mathbf{0 . 0 3}$ & 0.00 & 0.31 & 0.38 \\
9 & 99.65 & 0.00 & 1.87 & 0.03 & 0.00 & 0.31 & 0.38 \\
10 & 99.69 & 0.00 & 1.41 & 0.08 & 0.00 & 0.31 & 0.38 \\
& & & & & & & \\
\hline
\end{tabular}


Table IV

\section{Common Factors in US Dollar LIBOR and Swap rates}

Entries are the cumulative percentage of variation explained for each series by the first $K$ common factors in a three factor model, $K=1,2,3$. The last row reports the percentage of aggregate variance explained.

\begin{tabular}{lrrr}
\hline Factors & 1 & 2 & 3 \\
\hline $1 \mathrm{~m}$ & 58.94 & 95.70 & 98.09 \\
$2 \mathrm{~m}$ & 66.31 & 98.20 & 99.33 \\
$3 \mathrm{~m}$ & 72.94 & 98.66 & 99.06 \\
$6 \mathrm{~m}$ & 85.26 & 99.06 & 99.21 \\
$1 \mathrm{y}$ & 94.85 & 98.26 & 99.74 \\
$2 \mathrm{y}$ & 97.57 & 97.83 & 99.85 \\
$3 \mathrm{y}$ & 95.66 & 98.72 & 99.85 \\
$5 \mathrm{y}$ & 90.51 & 99.64 & 99.76 \\
$7 \mathrm{y}$ & 85.85 & 99.78 & 99.81 \\
$10 \mathrm{y}$ & 80.33 & 99.28 & 99.91 \\
$15 \mathrm{y}$ & 74.07 & 97.99 & 99.89 \\
$30 \mathrm{y}$ & 67.78 & 95.06 & \\
\hline Aggregate & & & 99.50 \\
\hline
\end{tabular}


Table V

Variance Decomposition on Implied Volatilities

Entries are the cumulative percentage variation explained for each implied volatility series by the three common factors in interest rates (in Panel A) and by one (Panel B), two (Panel C), three (Panel D) additional volatility factors. The maturity for each column represents the maturity of the underlying swap rate; the maturity for each row denotes the option maturity, all in years. The last row of each panel denotes averages across option maturities and the last column of each panel are averages across interest rate maturities. The bold numbers represent the aggregate percentage. The shaded cells highlight the series which have been explained by more than $90 \%$. Deeper shades imply that more factors are needed.

\begin{tabular}{rrrrrrrrr}
\hline Maturity & 1 & 2 & 3 & 4 & 5 & 7 & 10 & Average \\
\hline \multicolumn{7}{c}{ A. Three Common Factors in the Yield Curve } \\
\hline 0.1 & 63.18 & 62.88 & 60.82 & 59.90 & 59.59 & 56.54 & 52.35 & 59.32 \\
0.3 & 66.23 & 66.95 & 64.36 & 63.42 & 63.17 & 59.78 & 54.59 & 62.64 \\
0.5 & 71.29 & 69.11 & 67.35 & 66.52 & 66.77 & 63.77 & 58.19 & 66.14 \\
1 & 67.00 & 66.97 & 66.58 & 65.85 & 66.09 & 64.39 & 59.80 & 65.24 \\
2 & 58.27 & 60.73 & 61.30 & 61.00 & 60.30 & 55.94 & 49.16 & 58.10 \\
3 & 52.89 & 56.21 & 56.02 & 55.54 & 53.74 & 49.00 & 42.36 & 52.25 \\
4 & 47.98 & 51.83 & 50.56 & 48.54 & 46.66 & 42.97 & 39.22 & 46.82 \\
5 & 42.93 & 46.84 & 45.13 & 43.29 & 42.75 & 39.06 & 38.20 & 42.60 \\
7 & 46.89 & 48.94 & 46.44 & 44.15 & 41.27 & 37.72 & 37.04 & 43.21 \\
10 & 36.57 & 36.49 & 33.14 & 29.88 & 25.78 & 23.77 & 22.57 & 29.74 \\
Average & 55.32 & 56.69 & 55.17 & 53.81 & 52.61 & 49.30 & 45.35 & $\mathbf{5 9 . 4 8}$ \\
\hline
\end{tabular}

\begin{tabular}{rrrrrrrrr}
\hline \multicolumn{7}{c}{ B. One Additional Factor In Derivatives } \\
\hline 0.1 & 89.59 & 93.54 & 94.13 & 94.03 & 93.43 & 93.14 & 90.91 & 92.68 \\
0.3 & 91.27 & 96.26 & 96.62 & 96.88 & 96.58 & 95.77 & 93.09 & 95.21 \\
0.5 & 95.75 & 97.88 & 98.58 & 98.92 & 98.90 & 98.24 & 95.02 & 97.61 \\
1 & 93.62 & 94.18 & 93.63 & 92.75 & 91.91 & 89.85 & 86.01 & 91.71 \\
2 & 85.68 & 85.53 & 83.62 & 82.16 & 78.08 & 72.60 & 66.90 & 79.22 \\
3 & 78.48 & 78.12 & 76.13 & 72.59 & 68.30 & 61.95 & 55.52 & 70.16 \\
4 & 69.68 & 70.64 & 66.38 & 61.94 & 58.33 & 53.02 & 48.66 & 61.24 \\
5 & 61.13 & 61.33 & 57.86 & 54.25 & 51.63 & 46.52 & 45.19 & 53.99 \\
7 & 55.77 & 53.86 & 49.97 & 46.20 & 42.26 & 38.28 & 37.59 & 46.27 \\
10 & 38.83 & 36.90 & 33.17 & 29.96 & 26.31 & 24.59 & 23.26 & 30.43 \\
Average & 75.98 & 76.82 & 75.01 & 72.97 & 70.57 & 67.40 & 64.22 & $\mathbf{8 5 . 7 3}$ \\
& & & & & & & & \\
\hline
\end{tabular}


Table V

Variance Decomposition on Implied Volatilities (continued)

\begin{tabular}{rrrrrrrrr}
\hline Maturity & 1 & 2 & 3 & 4 & 5 & 7 & 10 & Average \\
\hline \multicolumn{7}{c}{ C. Two Additional Factors In Derivatives } \\
\hline 0.1 & 96.06 & 98.32 & 98.27 & 97.82 & 97.26 & 96.75 & 94.60 & 97.01 \\
0.3 & 96.69 & 99.10 & 99.22 & 99.00 & 98.64 & 97.60 & 94.87 & 97.87 \\
0.5 & 97.30 & 98.31 & 98.82 & 99.00 & 98.93 & 98.25 & 95.02 & 97.95 \\
1 & 94.57 & 96.30 & 97.13 & 97.97 & 98.52 & 98.25 & 95.48 & 96.89 \\
2 & 93.44 & 96.27 & 97.74 & 98.54 & 98.47 & 96.82 & 91.19 & 96.07 \\
3 & 93.59 & 97.36 & 98.49 & 98.68 & 98.16 & 95.40 & 87.91 & 95.65 \\
4 & 93.67 & 98.02 & 98.61 & 98.44 & 97.44 & 93.98 & 87.24 & 95.34 \\
5 & 93.07 & 98.29 & 98.44 & 97.89 & 96.80 & 92.22 & 86.31 & 94.72 \\
7 & 96.90 & 97.14 & 95.65 & 94.01 & 91.13 & 87.48 & 84.53 & 92.41 \\
10 & 85.66 & 78.85 & 75.02 & 71.76 & 66.15 & 62.31 & 56.79 & 70.93 \\
Average & 94.09 & 95.80 & 95.74 & 95.31 & 94.15 & 91.91 & 87.39 & $\mathbf{9 6 . 1 1}$ \\
\hline
\end{tabular}

D. Three Additional Factors In Derivatives

\begin{tabular}{rrrrrrrrr}
\hline 0.1 & 96.09 & 98.95 & 99.30 & 99.20 & 98.68 & 97.81 & 95.33 & 97.91 \\
0.3 & 96.98 & 99.27 & 99.47 & 99.28 & 98.86 & 97.67 & 94.87 & 98.06 \\
0.5 & 97.63 & 98.33 & 98.86 & 99.02 & 98.94 & 98.27 & 95.25 & 98.04 \\
1 & 95.45 & 96.96 & 97.60 & 98.37 & 98.83 & 98.66 & 95.99 & 97.41 \\
2 & 96.16 & 97.70 & 98.78 & 99.22 & 98.90 & 97.24 & 91.94 & 97.13 \\
3 & 97.25 & 99.01 & 99.49 & 99.32 & 98.53 & 95.80 & 88.73 & 96.88 \\
4 & 97.73 & 99.23 & 99.16 & 98.68 & 97.52 & 94.06 & 87.53 & 96.27 \\
5 & 96.96 & 98.88 & 98.62 & 97.90 & 96.87 & 92.30 & 86.31 & 95.41 \\
7 & 96.91 & 98.44 & 98.61 & 98.50 & 98.02 & 96.17 & 93.45 & 97.16 \\
10 & 92.55 & 94.55 & 95.17 & 95.64 & 95.78 & 95.52 & 91.71 & 94.42 \\
Average & 96.37 & 98.13 & 98.51 & 98.51 & 98.09 & 96.35 & 92.11 & $\mathbf{9 7 . 6 2}$ \\
& & & & & & & & \\
\hline
\end{tabular}


Table VI

\section{Correlations of the Factor Mimicking Portfolios with LIBOR and Swap Rates}

Entries are the correlations of the factor mimicking portfolios with LIBOR and swap rates. In the last two rows, we also report the correlations of the mimicking portfolios with, respectively, A spread and a butterfly portfolio. The spread is formed by going long one contract of the 30-year swap and shorting one contract of the one-month LIBOR. The butterfly is constructed by going long 1.6 contracts of the two-year swap and shorting one contract of the one-month LIBOR and 1.5 contracts of the 30-year swap. The bolded numbers represent the highest correlation between each mimicking factor and the interest rate portfolios.

\begin{tabular}{rrrrrrr}
\hline Factors & 1 & 2 & 3 & 4 & 5 & 6 \\
\hline $1 \mathrm{~m}$ & 0.77 & -0.61 & -0.15 & -0.02 & 0.01 & 0.01 \\
$2 \mathrm{~m}$ & 0.81 & -0.56 & -0.11 & -0.01 & 0.01 & -0.00 \\
$3 \mathrm{~m}$ & 0.85 & -0.51 & -0.06 & 0.01 & -0.00 & -0.02 \\
$6 \mathrm{~m}$ & 0.92 & -0.37 & 0.04 & 0.02 & -0.01 & 0.00 \\
$1 \mathrm{y}$ & 0.97 & -0.18 & 0.12 & 0.00 & -0.01 & 0.01 \\
$2 \mathrm{y}$ & $\mathbf{0 . 9 9}$ & 0.05 & 0.14 & -0.00 & -0.00 & 0.00 \\
$3 \mathrm{y}$ & 0.98 & 0.17 & 0.11 & -0.01 & 0.00 & -0.00 \\
$5 \mathrm{y}$ & 0.95 & 0.30 & 0.04 & -0.01 & 0.01 & -0.00 \\
$7 \mathrm{y}$ & 0.93 & 0.37 & -0.02 & -0.01 & 0.01 & -0.00 \\
$10 \mathrm{y}$ & 0.90 & 0.44 & -0.08 & -0.00 & 0.00 & -0.00 \\
$15 \mathrm{y}$ & 0.86 & 0.49 & -0.14 & 0.01 & -0.01 & -0.01 \\
$30 \mathrm{y}$ & 0.82 & 0.52 & -0.20 & 0.01 & -0.02 & 0.01 \\
\hline Spread & -0.03 & $\mathbf{0 . 9 8}$ & -0.02 & 0.03 & -0.02 & -0.00 \\
Butterfly & 0.00 & -0.00 & $\mathbf{0 . 9 9}$ & -0.01 & 0.02 & -0.04 \\
\hline
\end{tabular}




\section{Table VII}

\section{Correlations of the Mimicking Portfolios with the Implied Volatility Surface}

Entries are the correlations of the mimicking portfolios with the implied volatility surface. The maturity for each column represents the maturity of the underlying swap rates; the maturity for each row denotes the option maturity, all in years. The last row of each panel denotes averages across option maturities and the last column of each panel are averages across interest rate maturities. The bold numbers represent the grand average.

\begin{tabular}{lllllllll}
\hline Maturity & 1 & 2 & 3 & 4 & 5 & 7 & 10 & Average \\
\hline
\end{tabular}

A. Factor One: Interest Rate Level

\begin{tabular}{rrrrrrrrr}
\hline 0.1 & -0.53 & -0.51 & -0.51 & -0.52 & -0.53 & -0.54 & -0.55 & -0.53 \\
0.3 & -0.57 & -0.51 & -0.51 & -0.53 & -0.55 & -0.57 & -0.58 & -0.55 \\
0.5 & -0.58 & -0.50 & -0.51 & -0.53 & -0.56 & -0.57 & -0.59 & -0.55 \\
1.0 & -0.54 & -0.52 & -0.53 & -0.55 & -0.56 & -0.58 & -0.60 & -0.55 \\
2.0 & -0.44 & -0.49 & -0.51 & -0.53 & -0.54 & -0.55 & -0.55 & -0.52 \\
3.0 & -0.41 & -0.46 & -0.48 & -0.50 & -0.51 & -0.53 & -0.53 & -0.49 \\
4.0 & -0.38 & -0.43 & -0.45 & -0.47 & -0.48 & -0.51 & -0.54 & -0.47 \\
5.0 & -0.34 & -0.39 & -0.42 & -0.44 & -0.46 & -0.51 & -0.55 & -0.45 \\
7.0 & -0.32 & -0.38 & -0.40 & -0.43 & -0.45 & -0.49 & -0.53 & -0.43 \\
10.0 & -0.24 & -0.29 & -0.32 & -0.34 & -0.35 & -0.36 & -0.38 & -0.32 \\
Average & -0.44 & -0.45 & -0.46 & -0.48 & -0.50 & -0.52 & -0.54 & -0.48 \\
& & & & & & & & \\
\hline & & & B. Factor Two: Interest Rate Slope & \\
\hline 0.1 & 0.31 & 0.33 & 0.31 & 0.30 & 0.30 & 0.26 & 0.23 & 0.29 \\
0.3 & 0.29 & 0.34 & 0.31 & 0.29 & 0.28 & 0.25 & 0.21 & 0.28 \\
1.5 & 0.32 & 0.32 & 0.30 & 0.28 & 0.27 & 0.24 & 0.20 & 0.28 \\
2.0 & 0.27 & 0.24 & 0.22 & 0.21 & 0.21 & 0.20 & 0.19 & 0.22 \\
3.0 & 0.21 & 0.17 & 0.15 & 0.16 & 0.15 & 0.14 & 0.13 & 0.16 \\
4.0 & 0.18 & 0.15 & 0.15 & 0.14 & 0.14 & 0.12 & 0.08 & 0.14 \\
5.0 & 0.20 & 0.16 & 0.15 & 0.14 & 0.14 & 0.11 & 0.05 & 0.13 \\
7.0 & 0.18 & 0.11 & 0.15 & 0.15 & 0.14 & 0.09 & 0.01 & 0.13 \\
10.0 & 0.13 & 0.10 & 0.08 & 0.06 & 0.07 & 0.04 & -0.00 & 0.08 \\
Average & 0.23 & 0.21 & 0.19 & 0.18 & 0.18 & 0.15 & 0.11 & $\mathbf{0 . 1 8}$ \\
& & & & & & & & \\
\hline
\end{tabular}


Table VII

Correlations of the Mimicking Portfolios with the Implied Volatility Surface (continued)

\begin{tabular}{llllllllll}
\hline Maturity & 1 & 2 & 3 & 4 & 5 & 7 & 10 & Average
\end{tabular}

C. Factor Three: Interest Rate Curvature

\begin{tabular}{|c|c|c|c|c|c|c|c|c|}
\hline 0.1 & -0.50 & -0.51 & -0.51 & -0.49 & -0.48 & -0.45 & -0.41 & -0.48 \\
\hline 0.3 & -0.50 & -0.54 & -0.53 & -0.51 & -0.50 & -0.47 & -0.41 & -0.50 \\
\hline 0.5 & -0.53 & -0.58 & -0.57 & -0.55 & -0.53 & -0.50 & -0.44 & -0.53 \\
\hline 1.0 & -0.56 & -0.58 & -0.58 & -0.56 & -0.55 & -0.52 & -0.46 & -0.54 \\
\hline 2.0 & -0.58 & -0.58 & -0.57 & -0.55 & -0.53 & -0.48 & -0.41 & -0.53 \\
\hline 3.0 & -0.57 & -0.57 & -0.55 & -0.53 & -0.51 & -0.44 & -0.37 & -0.51 \\
\hline 4.0 & -0.55 & -0.55 & -0.53 & -0.50 & -0.46 & -0.39 & -0.32 & -0.47 \\
\hline 5.0 & -0.52 & -0.54 & -0.50 & -0.47 & -0.44 & -0.36 & -0.28 & -0.44 \\
\hline 7.0 & -0.58 & -0.58 & -0.54 & -0.50 & -0.45 & -0.37 & -0.30 & -0.47 \\
\hline 10.0 & -0.54 & -0.52 & -0.47 & -0.42 & -0.37 & -0.32 & -0.28 & -0.42 \\
\hline Average & -0.54 & -0.56 & -0.54 & -0.51 & -0.48 & -0.43 & -0.37 & -0.49 \\
\hline \multicolumn{9}{|c|}{ D. Derivative Factor One } \\
\hline 0.1 & 0.51 & 0.55 & 0.58 & 0.58 & 0.58 & 0.60 & 0.62 & 0.58 \\
\hline 0.3 & 0.50 & 0.54 & 0.57 & 0.58 & 0.58 & 0.60 & 0.62 & 0.57 \\
\hline 0.5 & 0.49 & 0.54 & 0.56 & 0.57 & 0.57 & 0.59 & 0.61 & 0.56 \\
\hline 1.0 & 0.52 & 0.52 & 0.52 & 0.52 & 0.51 & 0.50 & 0.51 & 0.51 \\
\hline 2.0 & 0.52 & 0.50 & 0.47 & 0.46 & 0.42 & 0.41 & 0.42 & 0.46 \\
\hline 3.0 & 0.51 & 0.47 & 0.45 & 0.41 & 0.38 & 0.36 & 0.36 & 0.42 \\
\hline 4.0 & 0.47 & 0.43 & 0.40 & 0.37 & 0.34 & 0.32 & 0.31 & 0.38 \\
\hline 5.0 & 0.43 & 0.38 & 0.36 & 0.33 & 0.30 & 0.27 & 0.26 & 0.33 \\
\hline 7.0 & 0.30 & 0.22 & 0.19 & 0.14 & 0.10 & 0.07 & 0.07 & 0.16 \\
\hline 10.0 & 0.15 & 0.06 & 0.02 & -0.03 & -0.07 & -0.09 & -0.08 & -0.01 \\
\hline Average & 0.44 & 0.42 & 0.41 & 0.39 & 0.37 & 0.36 & 0.37 & 0.40 \\
\hline
\end{tabular}




\section{Table VII}

\section{Correlations of the Mimicking Portfolios with the Implied Volatility Surface}

(Continued)

In Panel E, the "spread" portfolio is constructed by going long one unit of the one-month volatility and shorting four units of the five-year volatility. In Panel F, the "butterfly" portfolio is constructed by going long one unit of the one-month volatility, long two units of the ten-year volatility and shorting two units on the seven-year volatility.

\begin{tabular}{rrrrrrrrr}
\hline Maturity & 1 & 2 & 3 & 4 & 5 & 7 & 10 & Average \\
\hline \multicolumn{7}{c}{ E. Derivative Factor Two } \\
\hline 0.1 & 0.25 & 0.22 & 0.20 & 0.19 & 0.20 & 0.19 & 0.19 & 0.21 \\
0.3 & 0.23 & 0.17 & 0.16 & 0.15 & 0.14 & 0.14 & 0.13 & 0.16 \\
0.5 & 0.12 & 0.07 & 0.05 & 0.03 & 0.02 & 0.01 & 0.01 & 0.04 \\
1.0 & -0.10 & -0.15 & -0.19 & -0.23 & -0.26 & -0.29 & -0.31 & -0.22 \\
2.0 & -0.28 & -0.33 & -0.38 & -0.40 & -0.45 & -0.49 & -0.49 & -0.40 \\
3.0 & -0.39 & -0.44 & -0.47 & -0.51 & -0.55 & -0.58 & -0.57 & -0.50 \\
4.0 & -0.49 & -0.52 & -0.57 & -0.60 & -0.63 & -0.64 & -0.62 & -0.58 \\
5.0 & -0.57 & -0.61 & -0.64 & -0.66 & -0.67 & -0.68 & -0.64 & -0.64 \\
7.0 & -0.64 & -0.66 & -0.68 & -0.69 & -0.70 & -0.70 & -0.69 & -0.68 \\
10.0 & -0.68 & -0.65 & -0.65 & -0.65 & -0.63 & -0.61 & -0.58 & -0.64 \\
Spread & $\mathbf{0 . 9 1}$ & $\mathbf{0 . 9 4}$ & $\mathbf{0 . 9 5}$ & $\mathbf{0 . 9 5}$ & $\mathbf{0 . 9 5}$ & $\mathbf{0 . 9 2}$ & $\mathbf{0 . 8 7}$ & $\mathbf{0 . 9 3}$ \\
& & & & & & & & \\
\hline
\end{tabular}

F. Derivative Factor Three

\begin{tabular}{rrrrrrrrr}
\hline 0.1 & -0.02 & 0.08 & 0.10 & 0.12 & 0.12 & 0.10 & 0.09 & 0.08 \\
0.3 & -0.05 & 0.04 & 0.05 & 0.05 & 0.05 & 0.03 & 0.00 & 0.02 \\
0.5 & -0.06 & 0.01 & 0.02 & 0.01 & 0.01 & -0.01 & -0.05 & -0.01 \\
1.0 & -0.09 & -0.08 & -0.07 & -0.06 & -0.06 & -0.06 & -0.07 & -0.07 \\
2.0 & -0.17 & -0.12 & -0.10 & -0.08 & -0.07 & -0.06 & -0.09 & -0.10 \\
3.0 & -0.19 & -0.13 & -0.10 & -0.08 & -0.06 & -0.06 & -0.09 & -0.10 \\
4.0 & -0.20 & -0.11 & -0.07 & -0.05 & -0.03 & -0.03 & -0.05 & -0.08 \\
5.0 & -0.20 & -0.08 & -0.04 & -0.01 & 0.03 & 0.03 & 0.01 & -0.04 \\
7.0 & -0.01 & 0.11 & 0.17 & 0.21 & 0.26 & 0.29 & 0.30 & 0.19 \\
10.0 & 0.26 & 0.40 & 0.45 & 0.49 & 0.54 & 0.58 & 0.59 & 0.47 \\
Butterfly & $\mathbf{0 . 6 4}$ & $\mathbf{0 . 8 8}$ & $\mathbf{0 . 9 2}$ & $\mathbf{0 . 9 3}$ & $\mathbf{0 . 9 3}$ & $\mathbf{0 . 9 2}$ & $\mathbf{0 . 8 8}$ & $\mathbf{0 . 8 7}$ \\
& & & & & & & & \\
\hline
\end{tabular}


Table VIII

A $Z$-test for the Difference Between the Autocorrelations of the Explained Series and the Pricing Errors

Entries report the $Z$-test statistic of the null hypothesis that the explained part of the time series has the same first-order autocorrelation as the unexplained residuals under our $3+3$ factor model. The test has a normal distribution with mean zero and variance one. The first row denotes the LIBOR rate maturities in months. The second row reports the $Z$-test statistics for the LIBOR rates. The third and forth rows report the maturity (in years) and the $Z$-test statistics for swap rates. The bottom panel reports the tests for swaptions. The maturity for each column represents the maturity of the underlying swap rate; the maturity for each row denotes the option maturity, all in years.

\begin{tabular}{lrrrrrrr}
\hline LIBOR & 1 & 2 & 3 & 6 & 12 & & \\
& 30.26 & 39.81 & 31.44 & 30.12 & 57.59 & & \\
\hline \multirow{2}{*}{ Swap } & 2 & 3 & 5 & 7 & 10 & 15 & 30 \\
\hline & 50.73 & 43.54 & 35.38 & 26.13 & 29.75 & 30.27 & 27.34 \\
& & & & & & & \\
\hline \multirow{2}{*}{ Swaption } & 1 & 2 & 3 & 4 & 5 & 7 & 10 \\
0.1 & 19.08 & 23.85 & 23.53 & 22.12 & 16.60 & 15.22 & 12.90 \\
0.3 & 19.12 & 26.25 & 26.07 & 25.03 & 18.63 & 13.95 & 9.87 \\
0.5 & 18.89 & 16.24 & 19.66 & 24.96 & 26.66 & 24.11 & 18.37 \\
1 & 2.26 & 4.50 & 5.20 & 11.82 & 16.13 & 19.44 & 9.31 \\
2 & 2.68 & 9.99 & 13.95 & 22.71 & 20.45 & 11.81 & 3.14 \\
3 & 11.89 & 18.20 & 26.65 & 24.32 & 18.14 & 10.95 & 2.94 \\
4 & 19.50 & 26.22 & 27.57 & 20.66 & 17.62 & 8.47 & 6.24 \\
5 & 19.32 & 28.72 & 22.76 & 18.50 & 18.93 & 8.83 & 5.75 \\
7 & 26.90 & 25.68 & 28.07 & 28.40 & 29.01 & 22.27 & 15.64 \\
10 & 31.05 & 16.02 & 18.18 & 19.26 & 20.92 & 22.26 & 19.02 \\
& & & & & & & \\
\hline
\end{tabular}


Table IX

\section{Variance Explained and Number of Factors Tests For Differences}

The first row under each panel reports the percentage of variation explained by the first $K$ factors. The second to the fifth rows report the $t$-values and $p$-values of the unadjusted and adjusted tests $\left(\widehat{\Delta}\right.$ and $\widehat{\Delta}^{A}$ ) for the $K$ factor model against the $K+1$ alternative. The first three factors are tested on interest rates; the last three factors are tested on the regression residuals of the implied volatilities.

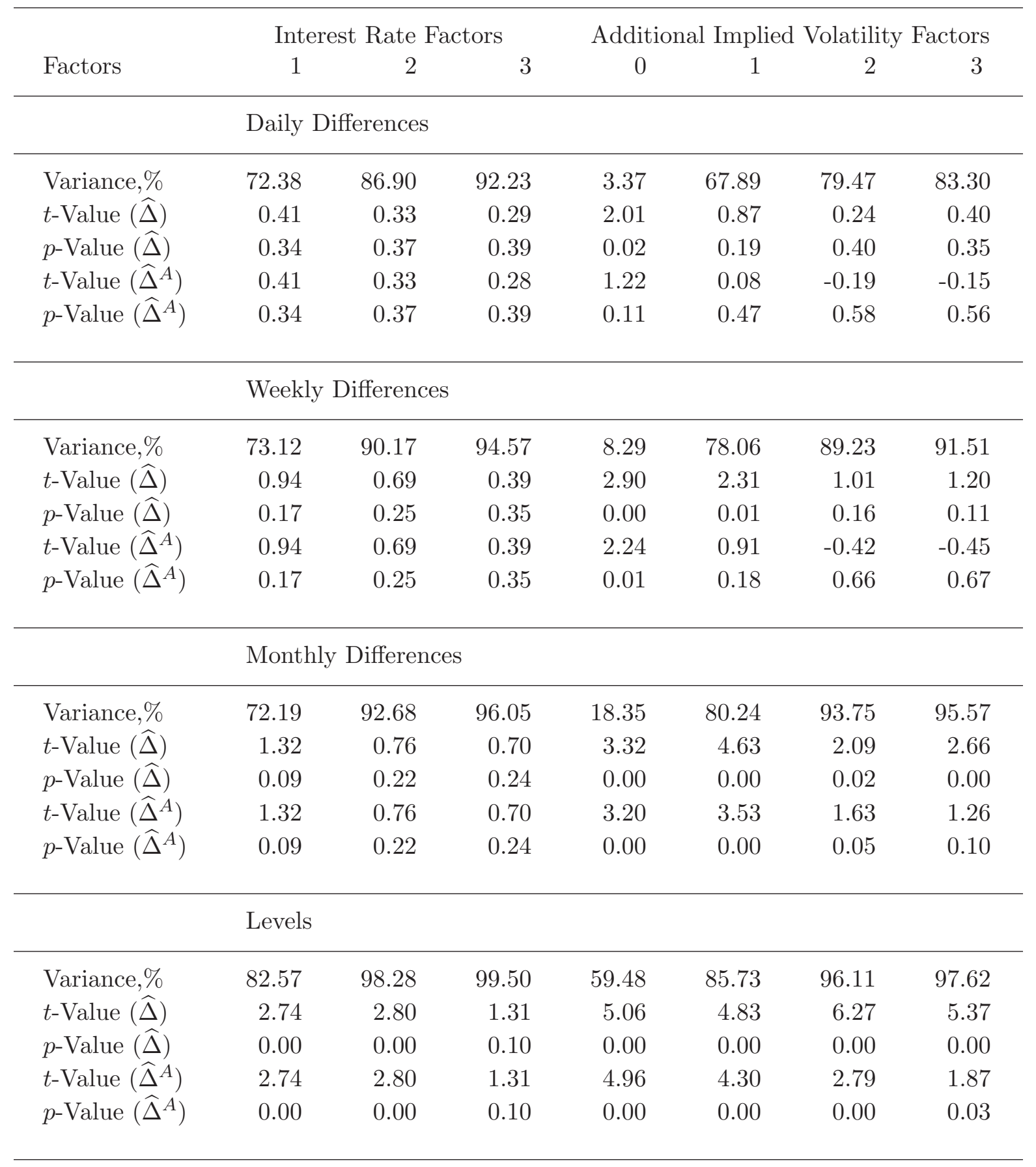


Table X

The Effects of Nonlinearities

Entries report the percentage of variation explained for each series by $K$ factors, where $K=$ $1,2, \cdots, 6 . r$ (the second column) denotes the series formed by the LIBOR and swap rates. $[r]^{n}$ denotes an expanded cross section that includes $r^{1}, \cdots, r^{n}$ and $1 / r, \cdots, 1 / r^{n}$. Thus, the third column, $[r]^{1}$ is formed by $r$ and $1 / r$; the fourth column, $[r]^{2}$ is formed by $r, r^{2}$ and $1 / r$ and $1 / r^{2}$, etc. The last column $[r, v]$ denotes the series that includes both the interest rates and the swaption implied volatilities. $N$ in the last row denotes the size of the cross section.

\begin{tabular}{|c|c|c|c|c|c|c|c|c|}
\hline$K$ & $r$ & {$[r]^{1}$} & {$[r]^{2}$} & {$[r]^{3}$} & {$[r]^{4}$} & {$[r]^{5}$} & {$[r]^{6}$} & {$[r, v]$} \\
\hline 1 & 81.79 & 81.60 & 82.06 & 82.62 & 83.20 & 83.77 & 84.33 & 84.35 \\
\hline 2 & 98.26 & 98.17 & 98.19 & 98.23 & 98.25 & 98.26 & 98.27 & 95.36 \\
\hline 3 & 99.48 & 99.39 & 99.41 & 99.45 & 99.47 & 99.49 & 99.50 & 97.02 \\
\hline 4 & 99.77 & 99.70 & 99.70 & 99.72 & 99.73 & 99.73 & 99.74 & 98.31 \\
\hline 5 & 99.88 & 99.82 & 99.82 & 99.83 & 99.83 & 99.83 & 99.83 & 98.97 \\
\hline 6 & 99.94 & 99.89 & 99.89 & 99.90 & 99.90 & 99.89 & 99.89 & 99.26 \\
\hline$N$ & 12 & 24 & 48 & 72 & 96 & 120 & 144 & 82 \\
\hline
\end{tabular}



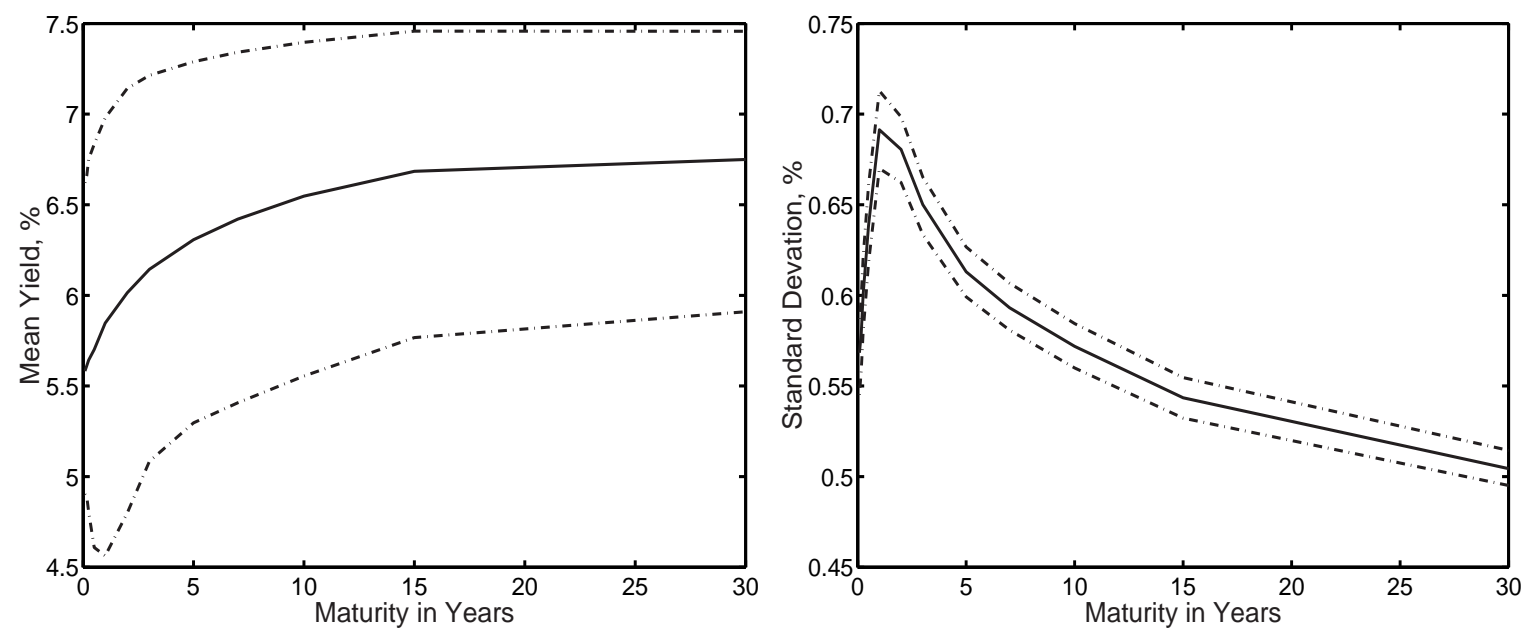

Figure 1. Mean and Standard Deviation of LIBOR and Swap Rates

Solid lines plot the term structure of the mean (left panel) and standard deviation (right panel) of the LIBOR and swap rates. Dashed lines represent the $5 \%$ and $95 \%$ quantiles. The quantiles are computed directly from the data for the mean in the left panel and from a 500 bootstrapped sample for the standard deviation in the right panel. The data are daily closing mid quotes from Lehman Brothers, ranging from October 30th, 1995 to July 23rd, 2001 (1434 observations).
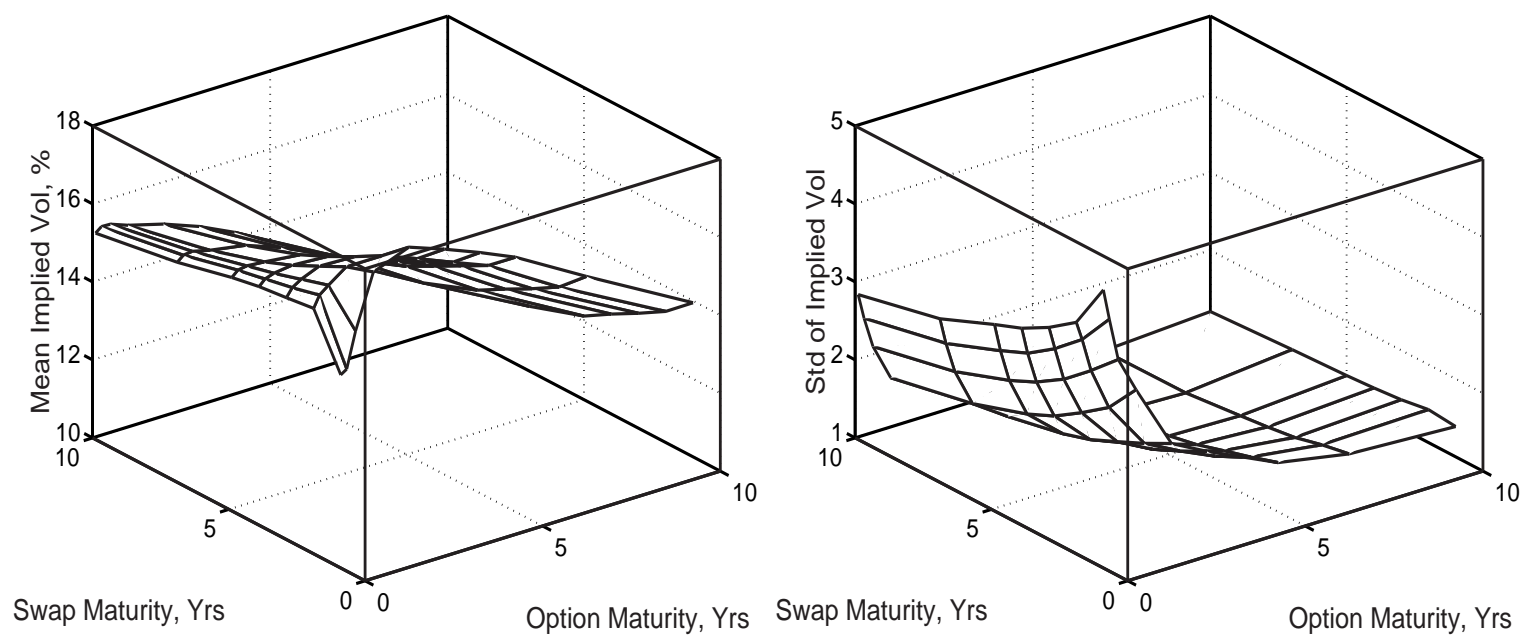

Figure 2. Mean and Standard Deviation of the Implied Volatility Surface The surface represents the mean (in the left panel) and standard deviation (in the right panel) estimates of the at-the-money Black implied volatility surface of swaptions. The data are daily closing quotes from Lehman Brothers, ranging from October 30th, 1995 to July 23rd, 2001 (1434 observations). 


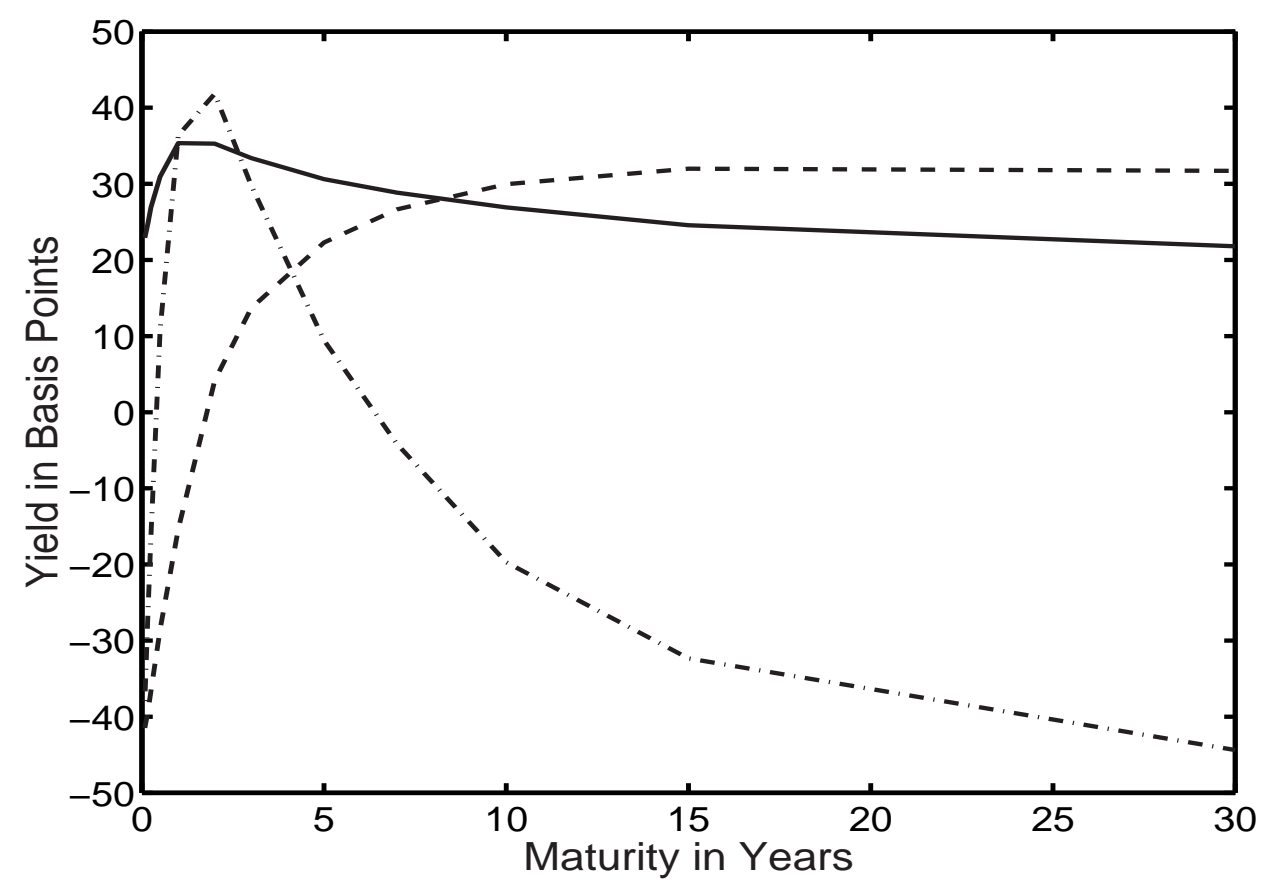

Figure 3. Factor Loadings on the Interest Rates

Lines depict the factor loadings (in basis points) on interest rates of different maturities. The solid line depicts the first factor, the dashed line depicts the second factor, and the dash-dotted line the third factor. 

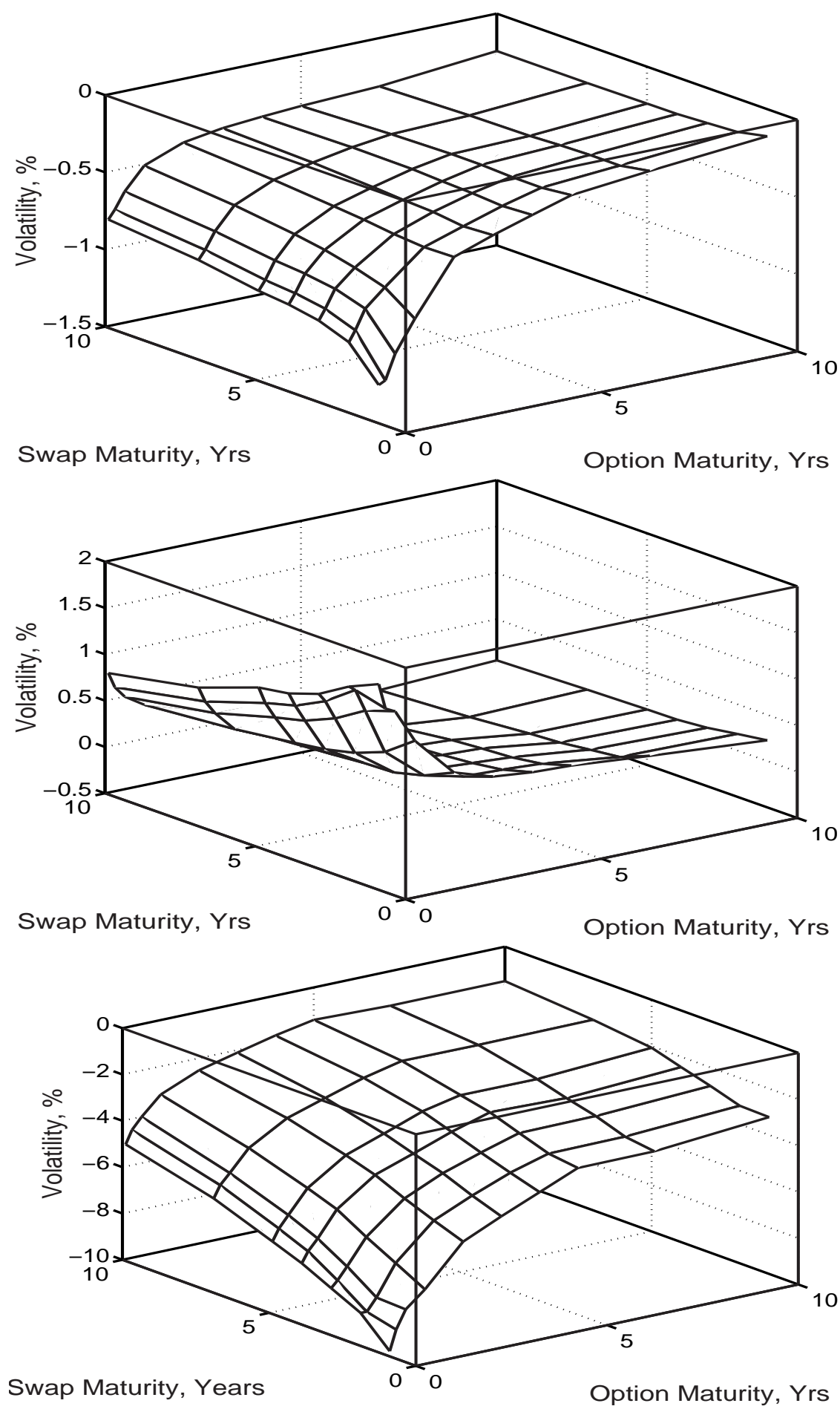

Figure 4. Loadings of Interest Rate Factors on the Implied Volatility Surface Lines depict the loadings (in percentage of the implied volatility) of the three interest rate common factors on the implied volatility surface of swaptions. The top panel depicts the impact of the level factor, the middle panel depicts the impact of the slope factor, and the bottom panel, the curvature factor. 

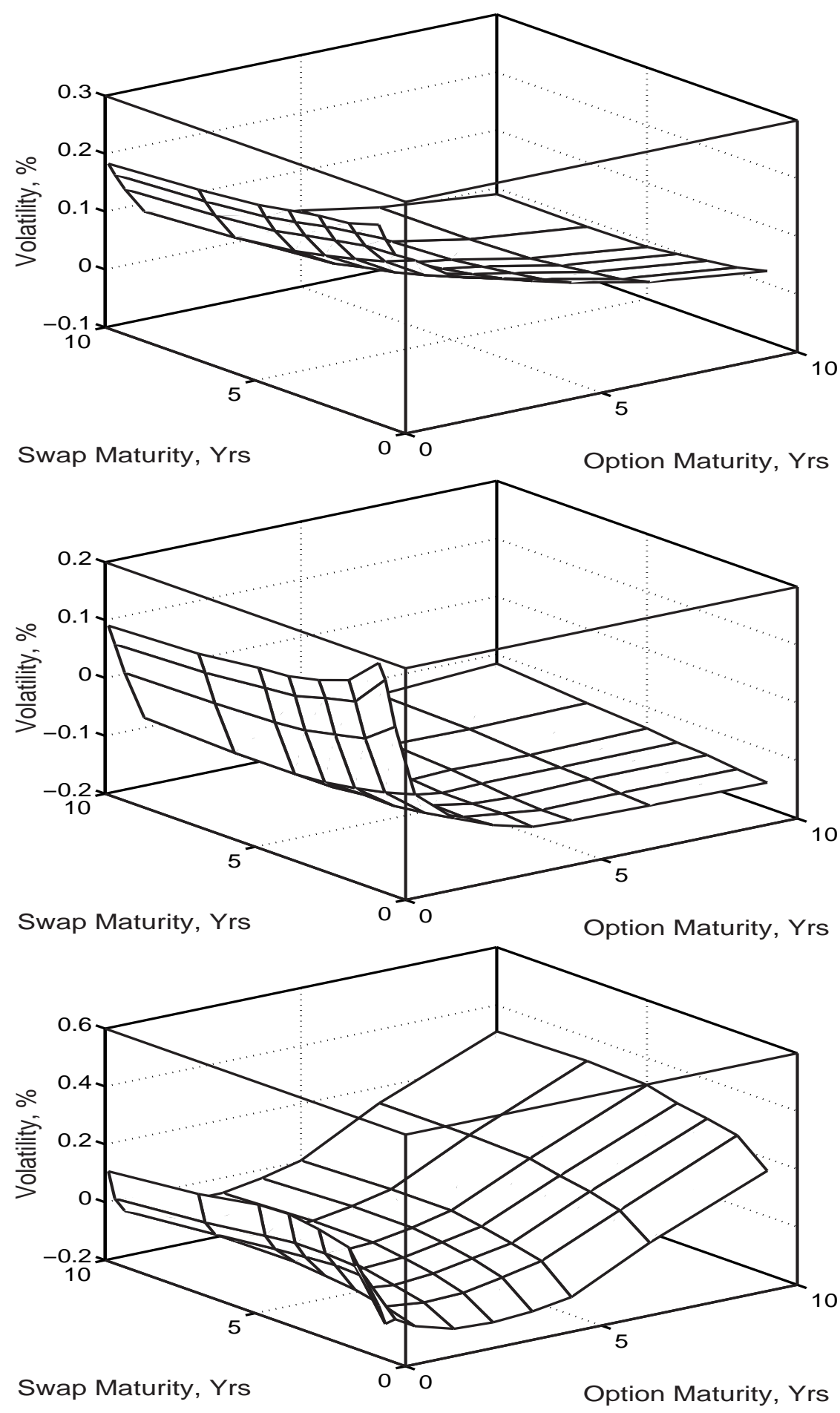

Figure 5. Loadings of Volatility Factors on the Implied Volatility Surface

Lines depict the loadings (in percentage of the implied volatility) of the the additional volatility factors on the implied volatility of swaptions. The top panel depicts the impact of the first volatility factor, the middle panel depicts the impact of the second volatility factor, and the bottom panel the third volatility factor. 